\title{
The Möbius function and continuous extensions of rotations
}

\section{J. Kułaga-Przymus ${ }^{1,2}$ - M. Lemańczyk ${ }^{2}$}

Received: 5 August 2014 / Accepted: 12 August 2015 / Published online: 1 September 2015

(C) The Author(s) 2015. This article is published with open access at Springerlink.com

\begin{abstract}
Let $f: \mathbb{T} \rightarrow \mathbb{R}$ be of class $C^{1+\delta}$ for some $\delta>0$ and let $c \in \mathbb{Z}$. We show that for a generic $\alpha \in \mathbb{R}$, the extension $T_{c, f}: \mathbb{T}^{2} \rightarrow \mathbb{T}^{2}$ of the irrational rotation $T x=x+\alpha$, given by $T_{c, f}(x, u)=(x+\alpha, u+c x+f(x))(\bmod 1)$ satisfies Sarnak's conjecture.
\end{abstract}

Keywords The Möbius function · Sarnak's conjecture $\cdot$ Skew products · Compact group extensions $\cdot$ Distal flows $\cdot$ Joinings

Mathematics Subject Classification $37 \mathrm{~A} 45 \cdot 11$ N37

\section{Contents}

1 Introduction . . . . . . . . . . . . . . . . . . . . . . . . . . . 554

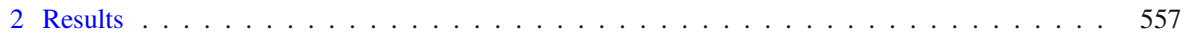

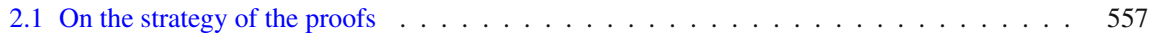

2.2 General remarks . . . . . . . . . . . . . . . . . . . . . . 558

Communicated by H. Bruin.

Research supported by Narodowe Centrum Nauki Grant DEC-2011/03/B/ST1/00407.

J. Kułaga-Przymus

joanna.kulaga@gmail.com

M. Lemańczyk

mlem@mat.umk.pl

1 Institute of Mathematics, Polish Academy of Sciences, Śniadeckich 8, 00-956 Warsaw, Poland

2 Faculty of Mathematics and Computer Science, Nicolaus Copernicus University, Chopina 12/18, 87-100 Toruń, Poland 
2.3 Continuous extensions of rational rotations . . . . . . . . . . . . . . . . . . 560

2.4 Affine case . . . . . . . . . . . . . . . . . . . . . . . . . 562

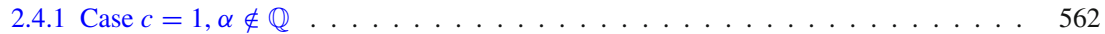

2.4 .2 Case $c=0, \alpha \notin \mathbb{Q} \ldots \ldots \ldots \ldots \ldots$. . . . . . . . . . . . . . . . . . . . . . . . . 566

2.5 Generic case: compact group extensions . . . . . . . . . . . . . . . . . . . . 567

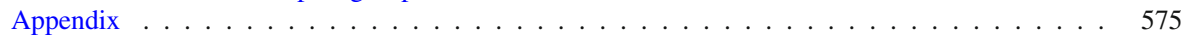

Notation and basic facts . . . . . . . . . . . . . . . . . . . . . 575

Compact group extensions: ergodicity and minimality . . . . . . . . . . . . . . . . . . 577

A remark on the KBSZ criterion for $T x=x+\alpha \ldots \ldots \ldots \ldots$. . . . . . . . . . 580

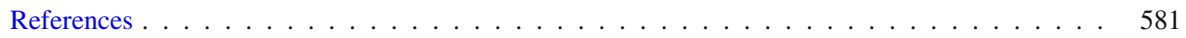

\section{Introduction}

Recall that the Möbius function $\mu: \mathbb{N} \rightarrow\{-1,0,1\}$ is a multiplicative function ${ }^{1}$ defined as $\boldsymbol{\mu}\left(p_{1} \cdot \ldots \cdot p_{k}\right)=(-1)^{k}$ for distinct prime numbers $p_{j}, \boldsymbol{\mu}(1)=1$ and 0 otherwise. Its importance is reflected in the fact that the prime number theorem ${ }^{2}$ is equivalent to the condition $\sum_{k \leq x} \boldsymbol{\mu}(k)=\mathrm{o}(x)$ and the Riemann hypothesis is equivalent to the condition $\sum_{k \leq x} \boldsymbol{\mu}(k)=\mathrm{O}_{\varepsilon}\left(x^{\frac{1}{2}+\varepsilon}\right.$ ) for any $\varepsilon>0$ (when $x \rightarrow$ $\infty$ ). The Möbius function appears to behave rather randomly and this statement was formalized in the following conjecture of Sarnak:

Conjecture 1 ([26]) Let $X$ be a compact metric space and let $T: X \rightarrow X$ be a homeomorphism of zero topological entropy. Let $x \in X, g \in C(X)$. Then

$$
\sum_{n \leq N} g\left(T^{n} x\right) \boldsymbol{\mu}(n)=\mathrm{o}(N)
$$

Whenever condition (1) is true for some $T$ for each $x \in X$ and each $g \in C(X)$, we say that Sarnak's conjecture holds for $T$ or that $T$ is disjoint with $\mu$. We will then write $T \perp \mu .{ }^{3}$

Sarnak's conjecture is known to hold in several situations, including rotations [9], nilsystems [15], horocycle flows [7], large class of rank one maps [2,6] and certain subclasses of dynamical systems generated by generalized Morse sequences [19], including the classical Thue-Morse system: $[1,5,8,14,16,24]$ and the dynamical system generated by the Rudin-Shapiro sequence [25].

One of the most fruitful tools used for proving disjointness with the Möbius function turns out to be the following orthogonality criterion of Katai-Bourgain-Sarnak-Ziegler (we will refer to it as KBSZ criterion).

Theorem 1.0.1 ([7,17]) Let $F: \mathbb{N} \rightarrow \mathbb{C}$ be a bounded sequence. Suppose that

$$
\sum_{n \leq N} F(r n) \overline{F(s n)}=\mathrm{o}(N)
$$

\footnotetext{
${ }^{1}$ Function $v: \mathbb{N} \rightarrow \mathbb{C}$ is said to be multiplicative if $v(m n)=v(m) v(n)$ for $n, m$ relatively prime.

2 Recall that the prime number theorem states that $\pi(x)=\frac{x}{\ln x}+\mathrm{o}\left(\frac{x}{\ln x}\right)$, where $\pi(x)$ is the number of primes less than $x$.

${ }^{3}$ Notice that it suffices to show that (1) holds for a linearly dense set of continuous functions to obtain disjointness with the Möbius function.
} 
for any pair of sufficiently large primes $r \neq s$. Then

$$
\sum_{n \leq N} F(n) v(n)=\mathrm{o}(N)
$$

for any multiplicative function $v$ with $|v| \leq 1$.

In order to use this theorem for proving Conjecture 1 for a given homeomorphism $T: X \rightarrow X$, one takes

$$
F(n):=g\left(T^{n} x\right) \quad \text { for } n \in \mathbb{Z}, x \in X \text { and } g \in C(X) \text {. }
$$

Notice that (2) for $F(n)$ given by (4) takes the form

$$
\sum_{n \leq N} g\left(T^{r n} x\right) \overline{g\left(T^{s n} x\right)}=\mathrm{o}(N)
$$

where

$$
\frac{1}{N} \sum_{n \leq N} g \otimes \bar{g}\left(\left(T^{r} \times T^{s}\right)^{n}(x, x)\right)=\int_{X^{2}} g \otimes \bar{g} d\left(\frac{1}{N} \sum_{n \leq N} \delta_{\left(T^{r} \times T^{s}\right)^{n}(x, x)}\right) .
$$

Thus, we are interested in the limit measures $\rho=\lim _{k \rightarrow \infty} \frac{1}{N_{k}} \sum_{n \leq N_{k}} \delta_{\left(T^{r} \times T^{s}\right)^{n}(x, x)}$ which are clearly $T^{r} \times T^{s}$-invariant. Therefore, to prove that $T \perp \mu$, it suffices to show that the following holds for $T$, for $r, s$ relatively prime:

(a) The ergodic components of $T^{r} \times T^{s}$ are pairwise disjoint closed sets filling up the whole space. ${ }^{4}$

(b) The ergodic components of $T^{r} \times T^{s}$ are uniquely ergodic (this implies that all points are generic for $T^{r} \times T^{s}$ for relevant invariant measures).

(c) There exists a linearly dense set of continuous functions $\mathcal{F} \subset C(X)$ such that for each $g \in \mathcal{F}, g \circ T \neq g$, we have $\int_{X^{2}} g \otimes \bar{g} d \rho=0$ for any $T^{r} \times T^{s}$-ergodic measure $\rho$, whenever $r, s$ are sufficiently large. ${ }^{5}$

We will use the strategy (a), (b), (c) to study disjointness with $\boldsymbol{\mu}$ in the following setting. Denote by $\mathbb{T}=\mathbb{R} / \mathbb{Z}=[0,1)$ the additive circle and consider

$$
\mathbb{T}^{2} \ni(x, y) \mapsto T_{c, f}(x, y):=(x+\alpha, y+c x+f(x)) \in \mathbb{T}^{2},
$$

\footnotetext{
4 The proof of the main result of the paper (Theorem 1.0.3 below) says also that the ergodic decomposition for $T^{r} \times T^{s}$ is the same as the decomposition into minimal components. This seems to be of independent interest. In case of continuous compact group extensions the existence of the decomposition into minimal components is guaranteed by a result of Auslander [4] and Ellis [10] on distal systems.

5 Notice that we do not aim to prove (5) for each $g \in C(X)$ (and each $x \in X$ ) -as a matter of fact (5) is not satisfied for some continuous functions already for an irrational rotation; we provide examples in "Appendix", see Proposition 2.5.17. Our aim is to prove (5) for a linearly dense set of $g \in C(X)$ (and each $x \in X$ ), as it implies that (1) holds for each $g \in C(X)$ (and each $x \in X$ ).
} 
where $c \in \mathbb{Z}$ and $c x+f(x)$ is a lift of a continuous circle map, i.e. $f: \mathbb{R} \rightarrow \mathbb{R}$ is continuous, periodic of period 1 , and $c$ is the degree of $f$. In other words, we consider continuous case of the classical Anzai skew product extensions of a rotation on the circle [3].

Liu and Sarnak in their recent paper [23] proved the following.

Theorem 1.0.2 ([23]) Let $c \in \mathbb{Z}$ and assume that $f: \mathbb{R} \rightarrow \mathbb{R}$ is an analytic periodic function of period 1. Assume additionally that $|\widehat{f}(m)| \gg e^{-\tau|m|}$ for some $\tau>0$. Then $T_{c, f} \perp \mu$.

The technical condition $|\widehat{f}(m)| \gg e^{-\tau|m|}$ on the Fourier transform seems to be necessary for the methods of [23] to work. On the other hand, there is no condition on $\alpha$. Moreover, for some $\alpha \mathrm{s}$, the result is obtained using Theorem 1.0.1. Under some additional assumptions, also a quantitative version [i.e. concerning the speed of convergence to zero in (1)] of Theorem 1.0.2 is proved in [23]. This is achieved by treating the problem in a more direct way than applying Theorem 1.0.1.

A natural question arises whether the assumptions on $f$ in Theorem 1.0.2 can be relaxed. Our main result (Theorem 1.0.3 below) provides a positive answer for each sufficiently smooth $f$, at the cost of reducing "for each $\alpha$ " in Theorem 1.0.2 to "for a generic $\alpha$ ". Hence, it can be viewed as complementary to Liu-Sarnak's result.

Theorem 1.0.3 Let $c \in \mathbb{Z}$ and assume that $f: \mathbb{R} \rightarrow \mathbb{R}$ is of class $C^{1+\delta}$ for some $\delta>0$, periodic of period 1 . Then, for a generic set of $\alpha$, we have $T_{c, f} \perp \mu$.

Remark 1.0.4 The question of whether an analogous result to Theorem 1.0.3 is true for $f$ which is only assumed to be continuous, remains open. ${ }^{6}$

Before we give the proof of Theorem 1.0.3, we first show that Conjecture 1 holds in the following two natural cases.

1. For an arbitrary continuous extension of a rational rotation (see Proposition 2.3.1), ${ }^{7}$

2. In the purely affine case (i.e. when $f=0$ ) for each $\alpha$.

More precisely, in case (2) we have:

Theorem 1.0.5 ([23]) Let $\alpha \in \mathbb{R}$ and $c \in \mathbb{Z}$. Let $\widetilde{T}: \mathbb{T}^{2} \rightarrow \mathbb{T}^{2}$ be given by $(x, y) \mapsto$ $(x+\alpha, c x+y+\gamma)$. Then $\widetilde{T} \perp \mu{ }^{8}$

Let us now describe how we check conditions (a), (b) and (c). Let $\alpha \notin \mathbb{Q}$ and let $T x=x+\alpha$. In either setting (purely affine or with a non-trivial perturbation) the base rotation $T^{r} \times T^{s}$ is the same. Its ergodic components are obtained by taking the partition of $\mathbb{T}^{2}$ into closed invariant sets $A_{c_{1}}=A_{c_{1}}^{r, s}:=\left\{\left(x, y+c_{1}\right) \in \mathbb{T}^{2}: s x=r y\right\}$, $c_{1} \in\left[0, \frac{1}{r}\right)$. In fact, these sets are at the same time the minimal components of $T^{r} \times T^{s}$ and they are uniquely ergodic. Thus, we are interested in the action of $\left(T_{c, f}\right)^{r} \times\left(T_{c, f}\right)^{s}$

\footnotetext{
6 We recall that under the continuity assumptions on $f$, even, it is open whether $f$ is not a quasi-coboundary for a generic set of $\alpha$.

7 Liu and Sarnak [23] prove analogous result for smooth $f$.

8 This result was first proved in a more general context in [23] by different methods.
} 
on the sets $I_{c_{1}}=I_{c_{1}}^{r, s}:=A_{c_{1}} \times \mathbb{T}^{2}$. It turns out that this is equivalent to dealing with extensions of $T$ by the following $\mathbb{T}^{2}$-valued cocycles:

$$
\psi_{c_{1}}(x)=\left(\psi^{(r)}(r x), \psi^{(s)}\left(s x+c_{1}\right)\right)
$$

where $\psi(x)=f(x)+c x$ and $c_{1} \in\left[0, \frac{1}{r}\right)$. The cocycle $\psi_{c_{1}}$ is ergodic if and only if

$$
e^{2 \pi i\left(A \psi^{(r)}(r x)+B \psi^{(s)}\left(s x+c_{1}\right)\right)} \text { is not a multiplicative coboundary }
$$

for $A, B \in \mathbb{Z}, A^{2}+B^{2} \neq 0$ (see Remark 2.5.10) ${ }^{9}$. This is the situation we aim for in course of the proof of Theorem 1.0.3. For a generic $\alpha$ we indeed obtain ergodicity of $\psi_{c_{1}}$ for all $c_{1}$-for the details see Corollary 2.5.8. Statement (b) follows from the fact that we deal with compact group extensions of rotations. Finally, we show that also (c) holds: given a non-trivial character $\chi \in \widehat{\mathbb{T}}^{2}$, we prove that for $r$ and $s$ relatively prime and large enough, the corresponding integrals of $\chi \otimes \bar{\chi}$ are zero. In case of Theorem 1.0.5, the problem is in a sense more delicate-some of the sets $I_{c_{1}}$ are too large to be the ergodic components and they need to be partitioned further into smaller subsets. This refined partition will be however satisfying (a). Condition (b) is proved in the same way as in Theorem 1.0.3. To prove that also (c) holds, we consider again $\chi \in \widehat{\mathbb{T}}^{2}$.

For the convenience of the reader, the necessary facts concerning cocycles are included in the "Appendix".

\section{Results}

\subsection{On the strategy of the proofs}

Our approach to proving disjointness with the Möbius function was described in (a), (b) and (c). We will now make some more comments on this method. Recall that in view of Theorem 1.0.1, what we want to prove is that for a linearly dense set $\mathcal{F}$ of $g \in C(X)$ and each $x \in X$, we have

$$
\sum_{n \leq N} g\left(T^{r n} x\right) \overline{g\left(T^{s n} x\right)}=\mathrm{o}(N),
$$

for distinct, sufficiently large prime numbers $r, s$. When this is realized through (a), (b) and (c), we prove more. Namely, for each $g \in \mathcal{F}$ and for sufficiently large primes $r \neq s$, the following holds for all $x, y \in X$ :

$$
\sum_{n \leq N} g\left(T^{r n} x\right) \overline{g\left(T^{s n} y\right)}=\mathrm{o}(N)
$$

(the condition on $r, s$ is independent of the choice of $x$ and $y$ ).

\footnotetext{
9 We identify $\mathbb{T}$ with the multiplicative circle $\mathbb{S}^{1}=\{z \in \mathbb{C}:|z|=1\}$. We will use both, the additive and the multiplicative notation, whichever is more convenient for us at the moment. In particular, $e^{2 \pi i x}$ is to be understood multiplicatively and $x$ in the exponent is to be understood additively.
} 
Remark 2.1.1 1. Let $k \geq 1$ and suppose that, for sufficiently large primes $r \neq s,(8)$ holds for $T^{k}$ and $g \in C(X)$, for all $x, y \in X$. Then

$$
\begin{aligned}
\sum_{n \leq N} g\left(T^{r(k n+j)} x\right) \overline{g\left(T^{s(k n+j)} x\right)} & =\sum_{n \leq N} g\left(\left(T^{k}\right)^{r n} T^{r j} x\right) \overline{g\left(\left(T^{k}\right)^{s n} T^{s j} x\right)} \\
& =\mathrm{o}(N) \quad \text { for } 0 \leq j<k
\end{aligned}
$$

It follows that for sufficiently large primes $r \neq s,(7)$ holds for $T$ and $g \in C(X)$ for all $x \in X$. In particular, in order to prove Sarnak's conjecture for $T$, it suffices to check conditions (a), (b) and (c) for $T^{k}$ for some $k \geq 1$.

2. Notice that whenever the conditions (a), (b) and (c) are satisfied for some homeomorphism $T$ then they are also satisfied for $T^{-1}$.

3. It is unclear how to prove directly that if $T^{k}$ for some $k \in \mathbb{Z} \backslash\{0\}$ is disjoint with $\mu$ then also $T$ is disjoint from $\mu$, or even to show that if the assumptions of the KBSZ criterion are satisfied for $T^{k}$ for some $k \in \mathbb{Z} \backslash\{0\}$ then they are satisfied for $T$.

Let now $T_{\varphi}: X \times \mathbb{T} \rightarrow X \times \mathbb{T}$ be a continuous circle group extension of a homeomorphism $T: X \rightarrow X$ by $\varphi: X \rightarrow \mathbb{T}$.

Remark 2.1.2 Suppose that $T_{\varphi} \perp \boldsymbol{\mu}$. Then for any $k \geq 1$ also $T_{k \varphi} \perp \boldsymbol{\mu}$ as $T_{k \varphi}$ is a topological factor of $T_{\varphi}$.

Remark 2.1.3 In the case of affine extensions of rotations, Remark 2.1.1 (1) and Remark 2.1.2 are complementary in the following sense. Let $T^{(\alpha)}$ stand for the rotation $T^{(\alpha)} x=x+\alpha$ and let $\psi(x)=x$. Then $\left(T_{\psi}^{\left(\frac{\alpha}{k}\right)}\right)^{k}=T_{k \psi+\frac{k-1}{2} \alpha}^{(\alpha)}$ and, by Remark 2.1.1 (1), the following implication holds:

$$
\text { ( (a), (b) and (c) hold for } \left.T_{k \psi+\frac{k-1}{2} \alpha}^{(\alpha)}\right) \Rightarrow T_{\psi}^{\left(\frac{\alpha}{k}\right)} \perp \boldsymbol{\mu} \text {. }
$$

\subsection{General remarks}

From now on, our assumption will be that

$$
r, s \geq 3 \text { are odd and relatively prime. }
$$

Let $\alpha \notin \mathbb{Q}$ and denote by $T: \mathbb{T} \rightarrow \mathbb{T}$ the irrational rotation $T x=x+\alpha$. For $c_{1} \in\left[0, \frac{1}{r}\right)$ let ${ }^{10}$

$$
I_{c_{1}}=I_{c_{1}}^{r, s}:=A_{c_{1}} \times \mathbb{T}^{2} \text {, where } A_{c_{1}}=A_{c_{1}}^{r, s}:=\left\{\left(x, y+c_{1}\right) \in \mathbb{T}^{2}: s x=r y\right\} .
$$

Lemma 2.2.1 The decomposition of $\mathbb{T}^{2}$ into minimal components of $T^{r} \times T^{s}$ consists of sets $A_{c_{1}}, c_{1} \in\left[0, \frac{1}{r}\right)$. It is the same as the ergodic decomposition. Moreover, $\left.\left(T^{r} \times T^{S}\right)\right|_{A_{c_{1}}}$ is topologically isomorphic to $T$. The isomorphism is given by

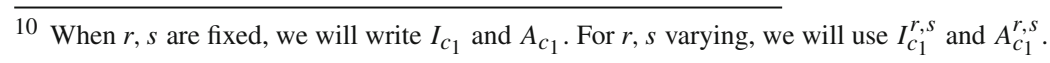




$$
W=W_{c_{1}}: A_{c_{1}} \rightarrow \mathbb{T}, W\left(x, y+c_{1}\right)=a x+b y,
$$

with $a, b \in \mathbb{Z}$ are such that ar $+b s=1$.

Proof Notice first that the sets $A_{c_{1}}$ are closed and invariant under $T^{r} \times T^{s}$ and $\bigcup_{c_{1} \in\left[0, \frac{1}{r}\right)} A_{c_{1}}=\mathbb{T}^{2}$. Fix $c_{1} \in\left[0, \frac{1}{r}\right)$, let $a, b \in \mathbb{Z}$ be such that $a r+b s=1$ and let $W$ be given by (10). Then $\left.W \circ\left(T^{r} \times T^{s}\right)\right|_{A_{c_{1}}}=T \circ W$. For $\left(x, y+c_{1}\right) \in A_{c_{1}}$, we have $r(a x+b y)=x$ and $s(a x+b y)=y$. Therefore, $W$ is bijective. Moreover, $W$ preserves the measure, as rotations are uniquely ergodic.

For a measurable function $\psi: \mathbb{T} \rightarrow \mathbb{T}$, let $\Psi: \mathbb{T}^{2} \rightarrow \mathbb{T}^{2}$ be given by

$$
\Psi(x, y)=\left(\psi^{(r)}(x), \psi^{(s)}(y)\right)
$$

Then clearly $\left(T_{\psi}\right)^{r} \times\left(T_{\psi}\right)^{s}$ is topologically isomorphic to $\left(T^{r} \times T^{s}\right)_{\Psi}$.

Lemma 2.2.2 For $c_{1} \in\left[0, \frac{1}{r}\right)$ the sets $I_{c_{1}}$ are invariant under $\left(T^{r} \times T^{s}\right)_{\Psi}$. Moreover, $\left.\left(T^{r} \times T^{s}\right)_{\Psi}\right|_{I_{c_{1}}}$ is topologically isomorphic to $T_{\psi_{c_{1}}}$, where $\psi_{c_{1}}(x)=$ $\left(\psi^{(r)}(r x), \psi^{(s)}\left(s x+c_{1}\right)\right)$. The isomorphism is given by

$$
V=V_{c_{1}}: I_{c_{1}} \rightarrow \mathbb{T}^{3}, V\left(x, y+c_{1}, u, v\right)=(a x+b y, u, v),
$$

with $a, b \in \mathbb{Z}$ such that $a r+b s=1$.

Proof Fix $c_{1} \in\left[0, \frac{1}{r}\right)$, let $a, b \in \mathbb{Z}$ be such that $a r+b s=1$ and let $V$ be given by (12). Then

$$
\begin{aligned}
V & \circ\left(T^{r} \times T^{s}\right)_{\Psi}\left(x, y+c_{1}, u, v\right) \\
& =V\left(x+r \alpha, y+s \alpha+c_{1}, u+\psi^{(r)}(x), v+\psi^{(s)}\left(y+c_{1}\right)\right) \\
& =\left(a x+b y+\alpha, u+\psi^{(r)}(x), v+\psi^{(s)}\left(y+c_{1}\right)\right)
\end{aligned}
$$

and

$$
\begin{aligned}
& T_{\psi_{c_{1}}} \circ V\left(x, y+c_{1}, u, v\right)=T_{\psi_{c_{1}}}(a x+b y, u, v) \\
& \quad=\left(a x+b y+\alpha, u+\psi^{(r)}(r(a x+b y)), v+\psi^{(s)}\left(s(a x+b y)+c_{1}\right)\right) .
\end{aligned}
$$

Moreover, $r(a x+b y)=x$ and $s(a x+b y)=y$, which completes the proof.

Remark 2.2.3 The inverse of $V_{c_{1}}: I_{c_{1}} \rightarrow \mathbb{T}^{3}$ is given by

$$
V_{c_{1}}^{-1}(t, u, v)=\left(r t, s t+c_{1}, u, v\right)
$$

Moreover, $a r t+b s t=t$ for $a, b \in \mathbb{Z}$ such that $a r+b s=1$. 


\subsection{Continuous extensions of rational rotations}

In this section we will prove the following:

Proposition 2.3.1 Let $p, q \in \mathbb{N}$, let $f: \mathbb{T} \rightarrow \mathbb{T}$ be continuous and let $R: \mathbb{T} \times \mathbb{T} \rightarrow$ $\mathbb{T} \times \mathbb{T}$ be given by $R(x, y)=\left(x+\frac{p}{q}, f(x)+y\right)$. Then $R \perp \mu$.

We will need an auxiliary lemma:

Lemma 2.3.2 Assume that $S(x, y)=(x, f(x)+y)$ with $f: \mathbb{T} \rightarrow \mathbb{T}$ continuous. Then for each $\left(x_{i}, y_{i}\right) \in \mathbb{T}^{2}, i=1,2, \chi \in \mathbb{T}^{2}$ we have

$$
\frac{1}{N} \sum_{n \leq N} \chi\left(S^{r n}\left(x_{1}, y_{1}\right)\right) \overline{\chi\left(S^{s n}\left(x_{2}, y_{2}\right)\right)} \rightarrow 0 \text { as } N \rightarrow \infty
$$

for sufficiently large prime numbers $r \neq s$, whenever $\chi \neq \eta \otimes \mathbb{1}_{\mathbb{T}}(\eta \in \widehat{\mathbb{T}})$ and $f\left(x_{1}\right) \notin \mathbb{Q}$ or $f\left(x_{2}\right) \notin \mathbb{Q}$.

Proof Note that for each $m \geq 1$,

$$
S^{m}(x, y)=(x, m f(x)+y)
$$

Let $\chi(x, y)=e^{2 \pi i(a x+b y)}$ for some $a, b \in \mathbb{Z}$, with $b \neq 0$ by assumption. Hence

$$
\begin{aligned}
& \frac{1}{N} \sum_{n \leq N} \chi\left(S^{r n}\left(x_{1}, y_{1}\right)\right) \overline{\chi\left(S^{s n}\left(x_{2}, y_{2}\right)\right)} \\
& \quad=e^{2 \pi i\left(a\left(x_{1}-x_{2}\right)+b\left(y_{1}-y_{2}\right)\right)} \frac{1}{N} \sum_{n \leq N} e^{2 \pi i b\left(r f\left(x_{1}\right)-s f\left(x_{2}\right)\right) n}
\end{aligned}
$$

If exactly one of the numbers $f\left(x_{1}\right)$ and $f\left(x_{2}\right)$ is irrational then the result follows from Weyl's criterion. If both $f\left(x_{1}\right)$ and $f\left(x_{2}\right)$ are irrational then there is at most one pair $(r, s)$ of relatively prime numbers such that $r f\left(x_{1}\right)-s f\left(x_{2}\right) \in \mathbb{Q}$ and we can again make use of Weyl's criterion, this time for $r, s$ sufficiently large.

Remark 2.3.3 Notice that the above proof says more. Namely, the convergence in (13) does not depend on $y_{1}, y_{2}$.

Proof (Proof of Proposition 2.3.1) We need to check (1) only for $g=\chi \in \widehat{\mathbb{T}^{2}}$. Suppose first that $\chi=\eta \otimes \mathbb{1}_{\mathbb{T}}$ with $\eta \in \widehat{\mathbb{T}}$. In this case, (1) follows from Sarnak's conjecture for finite systems.

Assume now that $\chi$ is not of the form $\eta \otimes \mathbb{1}_{\mathbb{T}}$ with $\eta \in \widehat{\mathbb{T}}$. Note that

$$
R^{q}(x, y)=\left(x, f_{q}(x)+y\right)
$$

where $f_{q}(x)=f(x)+f\left(x+\frac{1}{q}\right)+\cdots+f\left(x+\frac{q-1}{q}\right)$. Given $n \geq 1$, we take $n^{\prime}$ such that $n=q n^{\prime}+j$ with $0 \leq j<q$. Then, for each $\chi \in \widehat{\mathbb{T}^{2}}$, we have

$$
\chi\left(R^{r n}(x, y)\right) \overline{\chi\left(R^{s n}(x, y)\right)}=\chi\left(R^{q r n^{\prime}}\left(R^{r j}(x, y)\right)\right) \overline{\chi\left(R^{q s n^{\prime}}\left(R^{s j}(x, y)\right)\right)},
$$


where the first coordinates of the points $R^{r j}(x, y), R^{r j}(x, y)$ belong to the finite set $\left\{x, x+\frac{1}{q}, \ldots, x+\frac{q-1}{q}\right\}$ (hence do not depend on $r, s$ ). Hence, to obtain

$$
\frac{1}{N} \sum_{n \leq N} \chi\left(R^{r n}(x, y)\right) \overline{\chi\left(R^{s n}(x, y)\right)} \rightarrow 0
$$

for sufficiently large prime numbers $r \neq s$, we need to show that

$$
\frac{1}{N} \sum_{n^{\prime} \leq N / q} \chi\left(R^{q r n^{\prime}}\left(x_{1}, *\right)\right) \overline{\chi\left(R^{q s n^{\prime}}\left(x_{2}, *\right)\right)} \rightarrow 0
$$

for $x_{1}, x_{2} \in\left\{x, x+1 / q, \ldots, x+\frac{q-1}{q}\right\}$. It follows by Lemma 2.3.2, Remark 2.3.3 and (14), that (15) holds, provided that $f_{q}\left(x_{1}\right) \notin \mathbb{Q}$ or $f_{q}\left(x_{2}\right) \notin \mathbb{Q}$. Suppose now that $f_{q}(x+j r p / q), f_{q}(x+j s p / q) \in \mathbb{Q}$ for some $0 \leq j<q$. This is possible only if $f_{q}(x) \in \mathbb{Q}$ since $f_{q}(\cdot)$ is constant on the orbit of $x$ under the rotation $x \mapsto x+\frac{p}{q}$. Moreover, if $n=q n^{\prime}+j$ with $0 \leq j<q$ then

$$
f^{(n)}(x):=\sum_{i=0}^{n-1} f(x+i p / q)=f^{(j)}(x)+f^{\left(q n^{\prime}\right)}(x+j p / q)=f^{(j)}(x)+n^{\prime} f_{q}(x) .
$$

It follows that

$$
\begin{aligned}
\frac{1}{N} & \sum_{n \leq N} \chi\left(R^{n}(x, y)\right) \boldsymbol{\mu}(n) \\
& =\sum_{0 \leq j<q} \frac{1}{N} \sum_{n^{\prime} \leq N / q} \chi\left(x+\left(q n^{\prime}+j\right) p / q, f^{(j)}(x)+n^{\prime} f_{q}(x)+y\right) \boldsymbol{\mu}\left(q n^{\prime}+j\right) \\
& =\sum_{0 \leq j<q} \frac{1}{N} \sum_{n^{\prime} \leq N / q} \chi\left(x+j p / q, f^{(j)}(x)+n^{\prime} f_{q}(x)+y\right) \boldsymbol{\mu}\left(q n^{\prime}+j\right) \\
& =\sum_{0 \leq j<q} e^{2 \pi i\left(a(x+j p / q)+b f^{(j)}(x)\right)} \frac{1}{N} \sum_{n^{\prime} \leq N / q} e^{2 \pi i b f_{q}(x) n^{\prime}} \boldsymbol{\mu}\left(q n^{\prime}+j\right) \\
& =\sum_{0 \leq j<q} e^{2 \pi i\left(a(x+j p / q)+b f^{(j)}(x)\right)} \frac{1}{N} \sum_{n^{\prime} \leq N / q} e^{2 \pi i b \frac{c}{d} n^{\prime}} \boldsymbol{\mu}\left(q n^{\prime}+j\right),
\end{aligned}
$$

where $\chi(x, y)=e^{2 \pi i(a x+b y)}, f_{q}(x)=\frac{c}{d}$ with $a, b, c, d \in \mathbb{Z}, b \neq 0$ and $d>0$. By seting $n^{\prime}=d n^{\prime \prime}+k$ with $0 \leq k<d$, we obtain $q n^{\prime}+j=q d n^{\prime \prime}+(q k+j)$ and rewriting the sum to the form

$$
\sum_{0 \leq j<q} \sum_{0 \leq k<d} \frac{1}{N} \sum_{n^{\prime \prime} \leq N /(d q)} A_{j, k} \boldsymbol{\mu}\left(q d n^{\prime \prime}+k q+j\right),
$$

(1) again follows from Sarnak's conjecture for finite systems. 


\subsection{Affine case}

Recall that we are interested in the disjointness with the Möbius function of $(x, y) \mapsto$ $(x+\alpha, c x+y+\gamma)$, where $c \in \mathbb{Z}$ and $\alpha, \gamma \in \mathbb{R}$.

Remark 2.4.1 If $c \neq 0$, it follows immediately from Remark 2.1.2 that instead of $(x, y) \mapsto(x+\alpha, y+c x+\gamma)$, we can consider $(x, y) \mapsto\left(x+\alpha, y+x+\frac{\gamma}{c}\right)$. By Proposition 2.3.1, this shows that it suffices to consider only the cases $c=1$ and $c=0$, with $\alpha \notin \mathbb{Q}$.

\subsubsection{Case $c=1, \alpha \notin \mathbb{Q}$}

We will now deal with $(x, y) \mapsto(x+\alpha, y+x+\gamma)$, i.e. with $T_{\psi}$, where $\psi(x)=x+\gamma$. Let $\Psi$ be given by (11), i.e.

$$
\Psi(x, y)=\left(r x+\frac{(r-1) r}{2} \alpha+r \gamma, s y+\frac{(s-1) s}{2} \alpha+s \gamma\right) .
$$

Recall that given $r, s \in \mathbb{N}$ (odd and relatively prime) and $c_{1} \in\left[0, \frac{1}{r}\right.$ ), we have

$$
I_{c_{1}}=I_{c_{1}}^{r, s}=\left\{\left(x, y+c_{1}, u, v\right) \in \mathbb{T}^{4}: s x=r y\right\}
$$

For $c_{1}$ such that $r s\left((s-r) \gamma-r c_{1}\right) \in \alpha \mathbb{Q}+\mathbb{Q}$ and $c_{2} \in\left[0, \frac{1}{r^{2}}\right)$, define

$$
\begin{aligned}
J_{c_{1}, c_{2}}=J_{c_{1}, c_{2}}^{r, s} & :=\left\{\left(x, y+c_{1}, u, v+c_{2}\right) \in \mathbb{T}^{4}: s x=r y\right. \text { and } \\
l_{0} s^{2} u & \left.=l_{0} r^{2} v+\left(l_{0} r s \frac{r-s}{2}-k_{0}\right)(a x+b y)\right\}
\end{aligned}
$$

where $l_{0}=l_{0}^{r, s, c_{1}}$ is the smallest positive integer such that

$$
l_{0} r s\left((s-r) \gamma-r c_{1}\right) \in \alpha \mathbb{Z}+\mathbb{Z}
$$

and $k_{0}=k_{0}^{r, s, c_{1}} \in \mathbb{Z}$ is such that

$$
l_{0} r s\left((s-r) \gamma-r c_{1}\right)-k_{0} \alpha \in \mathbb{Z} .
$$

Lemma 2.4.2 For $c_{1} \in\left[0, \frac{1}{r}\right)$ the homeomorphism $T_{\psi_{c_{1}}}: \mathbb{T}^{3} \rightarrow \mathbb{T}^{3}$, where $\psi_{c_{1}}(x)=$ $\left(\psi^{(r)}(r x), \psi^{(s)}\left(s x+c_{1}\right)\right)$, is topologically isomorphic to $T_{\varphi_{c_{1}}}: \mathbb{T}^{3} \rightarrow \mathbb{T}^{3}$, where $\varphi_{c_{1}}: \mathbb{T} \rightarrow \mathbb{T}^{2}$ is given by $\varphi_{c_{1}}(x)=\left(r^{2} x+r \gamma, s^{2} x+s c_{1}+s \gamma\right)$. The isomorphism is given by

$$
U=U_{c_{1}}: \mathbb{T}^{3} \rightarrow \mathbb{T}^{3}, U(x, u, v)=\left(x, u-\frac{r(r-1)}{2} x, v-\frac{s(s-1)}{2} x\right)
$$


Proof We have

$$
\begin{aligned}
\psi_{c_{1}}(x) & =\left(\psi^{(r)}(r x), \psi^{(s)}\left(s x+c_{1}\right)\right) \\
& =\left(r^{2} x+\frac{r(r-1)}{2} \alpha+r \gamma, s\left(s x+c_{1}\right)+\frac{s(s-1)}{2} \alpha+s \gamma\right) .
\end{aligned}
$$

Since for $\theta(x)=\left(-\frac{r(r-1)}{2} x,-\frac{s(s-1)}{2} x\right)$ we have

$$
\left(\frac{r(r-1)}{2} \alpha, \frac{s(s-1)}{2} \alpha\right)=\theta(x)-\theta(x+\alpha),
$$

it follows that $U$ given by (19) is indeed the required isomorphism. Notice that $\theta$ is continuous, whence $U$ is a homeomorphism.

Proposition 2.4.3 The decomposition of $\mathbb{T}^{4}$ into minimal components for $\left(T^{r} \times T^{s}\right)_{\Psi}$ is the same as the decomposition into ergodic components. It consists of sets of the form $I_{c_{1}}$ for $c_{1} \notin \alpha \mathbb{Q}+\mathbb{Q}$ and $J_{c_{1}, c_{2}}$ for $c_{1} \in \alpha \mathbb{Q}+\mathbb{Q}$, where $c_{1} \in\left[0, \frac{1}{r}\right), c_{2} \in\left[0, \frac{1}{r^{2}}\right)$. Moreover, on each such component, $\left(T^{r} \times T^{s}\right)_{\Psi}$ is uniquely ergodic. In particular, each point in $\mathbb{T}^{4}$ is generic (for the relevant invariant measure).

Proof In view of Lemma 2.4.2, instead of $\left(T^{r} \times T^{s}\right)_{\Psi}$, we may consider $T_{\varphi_{c_{1}}}$, with $\varphi_{c_{1}}(x)=\left(r^{2} x+r \gamma, s^{2}+s c_{1}+s \gamma\right)$.

We will show first that the decomposition into minimal components for $\left(T^{r} \times\right.$ $\left.T^{s}\right)_{\Psi}$ is the same as the decomposition into ergodic components. By Remark 2.5.15, Proposition 2.5.9 and Proposition 2.5.12, it suffices to show that the equation

$$
\chi \circ \varphi_{c_{1}}=\xi-\xi \circ T
$$

has a measurable solution $\xi: \mathbb{T} \rightarrow \mathbb{T}$ for $\chi(u, v)=A u+B v$ for $A, B \in \mathbb{Z}$ with $A^{2}+B^{2} \neq 0$ if and only if it has a continuous one. We have

$$
\begin{aligned}
\chi \circ \varphi_{c_{1}}(x) & =\chi\left(r^{2} x+r \gamma, s^{2} x+s c_{1}+s \gamma\right) \\
& =\left(A r^{2}+B s^{2}\right) x+(A r+B s) \gamma+B s c_{1} .
\end{aligned}
$$

By [3], $\chi \circ \varphi_{c_{1}}$ can be a measurable coboundary only if

$$
A r^{2}+B s^{2}=0 \quad \text { and } \quad(A r+B s) \gamma+B s c_{1} \in \alpha \mathbb{Z}+\mathbb{Z}
$$

Then the solution to (20) is given by $\xi(x)=-k x$, where $k \in \mathbb{Z}$ is such that $(A r+$ $B s) \gamma+B s c_{1}-k \alpha \in \mathbb{Z}$. In particular, all measurable solutions to (20) are continuous.

We will now describe the ergodic (i.e. minimal) components of each $T_{\varphi_{c_{1}}}$ and show that they are uniquely ergodic. Suppose that $\chi \circ \varphi_{c_{1}}$ is a coboundary, i.e. for some measurable $\xi$, we have (20). It follows from (21) (recall that $r \neq s$ are coprime) that 
$r^{2} \mid B$ and $s^{2} \mid A$, and therefore $A=A^{\prime} s^{2}$ and $B=-A^{\prime} r^{2}$ for some $A^{\prime} \in \mathbb{Z}$. Hence, the second part of condition (21) takes the form

$$
A^{\prime} r s\left((s-r) \gamma-r c_{1}\right) \in \alpha \mathbb{Z}+\mathbb{Z}
$$

Having this in mind, we consider two cases:

1. $(s-r) \gamma-r c_{1} \notin \mathbb{Q} \alpha+\mathbb{Q}$,

2. $(s-r) \gamma-r c_{1} \in \mathbb{Q} \alpha+\mathbb{Q}$.

In case (1), it follows immediately from the first part of the proof that $\left.\left(T^{r} \times T^{s}\right)_{\Psi}\right|_{I_{c_{1}}}$ is ergodic and minimal. The unique ergodicity of $\left.\left(T^{r} \times T^{s}\right)_{\Psi}\right|_{I_{c_{1}}}$ follows from Proposition 2.5.16. Consider now case (2). We will describe characters $\chi$, such that (20) has a (measurable and continuous) solution. In view of and (17) and (22), $A^{\prime}=a l_{0}$ for some $a \in \mathbb{Z}$. Therefore, a measurable solution $\xi: \mathbb{T} \rightarrow \mathbb{T}$ to (20) exists precisely for the characters $\chi$ of $\mathbb{T}^{2}$ of the form

$$
\chi(u, v)=a l_{0} s^{2} u-a l_{0} r^{2} v \quad \text { for } a \in \mathbb{Z}
$$

Denote the set of such characters by $\Gamma=\Gamma_{\text {top }}$. It is easy to see that

$$
\text { ann } \Gamma=\left\{(u, v) \in \mathbb{T}^{2}: l_{0} s^{2} u=l_{0} r^{2} v\right\}
$$

We claim that

$$
\widetilde{J}_{c_{1}, c_{2}}^{r, s}:=\left\{\left(t, u, v+c_{2}\right) \in \mathbb{T}^{3}: l_{0} s^{2} u=l_{0} r^{2} v-k_{0} t\right\} \quad \text { for } c_{2} \in\left[0,1 / r^{2}\right)
$$

are minimal components of $T_{\varphi_{c_{1}}}$. Indeed:

- Each $\widetilde{J}_{c_{1}, c_{2}}^{r, s}$ is closed,

- By (18), each $\widetilde{J}_{c_{1}, c_{2}}^{r, s}$ is $T_{\varphi_{c_{1}}}$-invariant,

- $\bigcup_{c_{2}} \widetilde{J}_{c_{1}, c_{2}}^{r, s}=\mathbb{T}^{3}$ (notice that the projection of $\widetilde{J}_{c_{1}, c_{2}}^{r, s}$ onto the first two coordinates is equal to $\mathbb{T}^{2}$ ),

- $(u, v) \in$ ann $\Gamma$ if and only if $\widetilde{J}_{c_{1}, c_{2}}^{r, s}+(0, u, v)=\widetilde{J}_{c_{1}, c_{2}}^{r, s}$

(see Proposition 2.5.12). Unique ergodicity follows, as in the previous case, from Proposition 2.5.16.

To find the minimal component corresponding to each of the sets $\widetilde{J}_{c_{1}, c_{2}}^{r, s}$ described above, we need to find the preimage of $\widetilde{J}_{c_{1}, c_{2}}^{r, s}$ via $U_{c_{1}} \circ V_{c_{1}}$. We have

$$
\begin{aligned}
U_{c_{1}}^{-1}\left(\widetilde{J}_{c_{1}, c_{2}}^{r, s}\right) & =\left\{\left(t, u, v+c_{2}\right): l_{0} s^{2}\left(u-\frac{r(r-1)}{2} t\right)=l_{0} r^{2}\left(v-\frac{s(s-1)}{2} t\right)-k_{0} t\right\} \\
& =\left\{\left(t, u, v+c_{2}\right): l_{0} s^{2} u=l_{0} r^{2} v+\left(l_{0} r s \frac{r-s}{2}-k_{0}\right) t\right\},
\end{aligned}
$$


whence, by Remark 2.2.3,

$$
\begin{aligned}
\left(U_{c_{1}} V_{c_{1}}\right)^{-1}\left(\widetilde{J}_{c_{1}, c_{2}}^{r, s}\right)= & \left\{\left(r t, s t+c_{1}, u, v+c_{2}\right):\right. \\
& \left.l_{0} s^{2} u=l_{0} r^{2} v+\left(l_{0} r s \frac{r-s}{2}-k_{0}\right) t\right\} \\
= & \left\{\left(x, y+c_{1}, u, v+c_{2}\right): s x=r y\right. \text { and } \\
& \left.l_{0} s^{2} u=l_{0} r^{2} v+\left(l_{0} r s \frac{r-s}{2}-k_{0}\right)(a x+b y)\right\} .
\end{aligned}
$$

Therefore $\left(U_{c_{1}} V_{c_{1}}\right)^{-1}\left(\widetilde{J}_{c_{1}, c_{2}}^{r, s}\right)=J_{c_{1}, c_{2}}^{r, s}$, which completes the proof.

Remark 2.4.4 The sets $I_{c_{1}}^{r, s}$ are translates of $I_{0}^{r, s}$, which is a subgroup of $\mathbb{T}^{4}$. Therefore, the conditional measures given by the ergodic decomposition of $\left(T^{r} \times T^{s}\right) \Psi$, which are supported on the sets $I_{c_{1}}$ for $c_{1}$ such that $(s-r) \gamma-r c_{1} \notin \mathbb{Q} \alpha+\mathbb{Q}$, are translates of Haar measure on $I_{0}^{r, s}$.

For $c_{1}$ with $(s-r) \gamma-r c_{1} \in \mathbb{Q} \alpha+\mathbb{Q}$, the sets $\widetilde{J}_{c_{1}, c_{2}}^{r, s}$ are translates of $\widetilde{J}_{c_{1}, 0}^{r, s}$, which is a subgroup of $\mathbb{T}^{3}$. As before, the conditional measures given by the ergodic decomposition of $T_{\varphi_{c_{1}}}$, are translates of Haar measure on $\widetilde{J}_{c_{1}, 0}^{r, s}$. Notice that $V_{c_{1}}$ carries a translate of Haar measure to Haar measure. Therefore, also the conditional measures given by the ergodic decomposition of $\left(T^{r} \times T^{s}\right)_{\Psi}$, which are supported on the sets $J_{c_{1}, c_{2}}^{r, s}$, are translates of the corresponding Haar measures.

Remark 2.4.5 Let $G$ be a compact Abelian group. Let $\chi \in \widehat{G}$ and let $H=\operatorname{ker} \chi$. Then $\chi$ is constant on each coset of $H$. Moreover, if $x+H \neq y+H$ then $\chi(x+H) \neq$ $\chi(y+H)$.

Remark 2.4.6 Let $\chi \in \widehat{G}$ and let $H \subset G$ be a subgroup. Then the integral of $\chi$ with respect to Haar measure on each coset of $H$ is zero if and only if $\left.\chi\right|_{H}$ is not constant.

Lemma 2.4.7 Let $G$ be a compact metric Abelian group. Let $\chi \in \widehat{G}$. Then there exists $\delta>0$ such that whenever $\left\{y_{1}, \ldots, y_{n}\right\} \subset G$ is a $\delta$-net and $\chi\left(y_{i}\right)=1$ for $i=1, \ldots, n$ then $\chi \equiv 1$.

Proof Let $d$ stand for the metric on $G$. Since $\chi$ is uniformly continuous, there exists $\delta>0$ such that $d(x, y)<\delta$ implies $|\chi(x)-\chi(y)|<\frac{1}{4}$. Since $\left\{y_{1}, \ldots, y_{n}\right\}$ is a $\delta$-net, it follows that $|\chi(G)-1|<\frac{1}{2}$. This is however possible only when $\chi \equiv 1$, as $\chi(G)$ is a closed subgroup of $\mathbb{S}^{1}$.

Lemma 2.4.8 Let $\chi \in \widehat{\mathbb{T}}^{4}$ be a non-trivial character. If $r, s \in \mathbb{N}$ are large enough then $\left.\chi\right|_{I_{c_{1}}}$ is not constant for $c_{1} \in\left[0, \frac{1}{r}\right)$.

Proof Fix $1 \not \equiv \chi \in \widehat{\mathbb{T}}^{4}$ and let $\delta>0$ be as in Lemma 2.4.7. In view of Lemma 2.4.7, we need to show that whenever $r, s$ are large enough then there exists a $\delta$-net of $\mathbb{T}^{4}$ contained in $I_{c_{1}}^{r, s}$ for $c_{1} \in\left[0, \frac{1}{r}\right)$. Since $I_{c_{1}}^{r, s}$ is a translate of $I_{0}^{r, s}$ and the third and fourth coordinate in $I_{0}$ is arbitrary, it suffices to prove that for $r, s$ sufficiently large we can always find a $\delta$-net of $\mathbb{T}^{2}$ contained in the set $A_{0}^{r, s}$. To complete the proof it suffices to consider sets of the form $\left\{\left(\frac{i}{s}, \frac{j}{r}\right): 0 \leq i<s, 0 \leq j<r\right\}$. 
Lemma 2.4.9 Let $\chi \in \widehat{\mathbb{T}}^{4}$ be a non-trivial character. If $r, s \in \mathbb{N}$ are large enough then $\left.\chi\right|_{J_{c_{1}, c_{2}}^{r, s}}$ is not constant for $c_{1} \in\left[0, \frac{1}{r}\right)$ such that $c_{1} \in \alpha \mathbb{Q}+\mathbb{Q}$ and $c_{2} \in\left[0, \frac{1}{r^{2}}\right)$.

Proof Fix $1 \not \equiv \chi \in \widehat{\mathbb{T}}^{4}$ and let $\delta>0$ be as in Lemma 2.4.7. Since $J_{c_{1}, c_{2}}^{r, s}$ is a translation of $J_{c_{1}, 0}^{r, s}$, by Lemma 2.4.7, it suffices to prove that for $r, s$ sufficiently large there exists a $\delta$-net of $\mathbb{T}^{4}$ in $J_{c_{1}, 0}^{r, s}$.

Notice first that the projection of $J_{c_{1}, 0}^{r, s}$ onto the first two coordinates is equal to $A_{c_{1}}^{r, s}$. Indeed, for any $z \in \mathbb{T}$ the equation $l_{0} s^{2} u=l_{0} r^{2} v+z$ has a solution $(u, v)$ as $\mathbb{T}$ is an infinitely divisible group. By the proof of the previous lemma, we can find a $\delta$-net of $\mathbb{T}^{2}$

$$
\left\{\left(x_{i}, y_{j}\right): 0 \leq i, j<n\right\} \subset A_{c_{1}}^{r, s} .
$$

Moreover, by the infinite divisibility of $\mathbb{T}$, for each $(i, j)$ there exist $u_{i, j}, v_{i, j}$ such that $\left(x_{i}, y_{j}, u_{i, j}, v_{i, j}\right) \in J_{c_{1}, 0}^{r, s}$. Moreover, for $0 \leq a, b<l_{0} s^{2}$

$$
\left(x_{i}, y_{j}, u_{i, j}+\frac{a}{l_{0} s^{2}}, v_{i, j}+\frac{b}{l_{0} s^{2}}\right) \in J_{c_{1}, 0}^{r, s} .
$$

Therefore, whenever $r, s$ are sufficiently large, the set

$$
\left\{\left(x_{i}, y_{j}, u_{i, j}+\frac{a}{l_{0} s^{2}}, v_{i, j}+\frac{b}{l_{0} s^{2}}\right): 0 \leq i, j<n, 0 \leq a, b<l_{0} s^{2}\right\}
$$

is the required $\delta$-net. ${ }^{11}$ This completes the proof.

Proposition 2.4.10 For any $1 \not \equiv \chi \in \widehat{\mathbb{T}}^{4}$ whenever $r, s \in \mathbb{N}$ are odd, relatively prime and large enough then $\int_{I} \chi d \lambda_{I}=0$ for each ergodic component of $\left(T^{r} \times T^{s}\right)_{\Psi}$, where $\lambda_{I}$ is the relevant invariant measure.

Proof The assertion follows immediately from Proposition 2.4.3, Lemmas 2.4.8 and 2.4.9.

Proof of Theorem 1.0.5 in case $c=1, \alpha \notin \mathbb{Q}$. We only need to check that (a), (b), (c) hold. Conditions (a) and (b) follow from Proposition 2.4.3. Moreover, by Proposition 2.4.10, also condition (c) is satisfied (we take $\mathcal{F}=\widehat{\mathbb{T}}^{2}$ ).

\subsubsection{Case $c=0, \alpha \notin \mathbb{Q}$}

Proof of Theorem 1.0.5 in case $c=0, \alpha \notin \mathbb{Q}$. We have $T_{\psi}(x, u)=(x+\alpha, u+\gamma)$, i.e. $T_{\psi}$ is a rotation on $\mathbb{T}^{2}$. The decomposition into minimal components of $\left(T^{r} \times T^{s}\right)_{\Psi}$ consists of the cosets of $I_{0}=I_{0}^{r, s}=\left\{(x, y, 0,0) \in \mathbb{T}^{4}: s x=r y\right\}$. They are at the same the ergodic components which are moreover uniquely ergodic, and we conclude as previously.

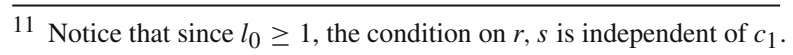




\subsection{Generic case: compact group extensions}

Let $f: \mathbb{R} \rightarrow \mathbb{R}$ (periodic of period 1 ) be in $L^{2}(\mathbb{T})$. Denote by

$$
f(x)=\sum_{n \in \mathbb{Z}} \widehat{f}(n) e^{2 \pi i n x}
$$

the Fourier expansion of $f$. Recall that our goal is to prove disjointness of

$$
\mathbb{T}^{2} \ni(x, y) \mapsto(x+\alpha, y+c x+f(x)) \in \mathbb{T}^{2},
$$

with the Möbius function $\boldsymbol{\mu}$ for a generic set of $\alpha$ (under some additional assumptions on $f$ ).

Recall the following result.

Theorem 2.5.1 $([18,21])^{12}$ Suppose that $f(x)=\sum_{n \in \mathbb{Z}} \widehat{f}(n) e^{2 \pi i n x}$ is in $C^{1+\delta}(\mathbb{T})$ for some $\delta>0$, and has zero mean. Denote by $T: \mathbb{T} \rightarrow \mathbb{T}$ the irrational rotation $x \mapsto x+\alpha$. Assume that for a sequence $\left(p_{n} / q_{n}\right)_{n \in \mathbb{N}}$ of rational numbers we have

$$
\frac{\left|\widehat{f}\left(q_{n}\right)\right|}{\sum_{k \geq 1}\left|\widehat{f}\left(k q_{n}\right)\right|}>c>0
$$

and

$$
\frac{\left|\alpha-\frac{p_{n}}{q_{n}}\right| q_{n}}{\left|\widehat{f}\left(q_{n}\right)\right|} \rightarrow 0 .
$$

Then, for each $\lambda \in \mathbb{S}^{1}$, the cocycle $\lambda e^{2 \pi i f(\cdot)}$ is not a T-coboundary.

We will now prove a modified version of the above theorem. It will be our main tool in course of the proof of Theorem 1.0.3.

Theorem 2.5.2 Suppose that $f(x)=\sum_{n \in \mathbb{Z}} \widehat{f}(n) e^{2 \pi i n x}$ is in $C^{1+\delta}(\mathbb{T})$ for some $\delta>$ 0 , and has zero mean. Denote by $T: \mathbb{T} \rightarrow \mathbb{T}$ the irrational rotation $x \mapsto x+\alpha$. Let $r, s \in \mathbb{N}$ be relatively prime. Assume that $\left(p_{n} / q_{n}\right)_{n \in \mathbb{N}}$ is a subsequence of convergents of $\alpha$ in its continued fraction expansion such that

$$
\begin{gathered}
\frac{\left|\widehat{f}\left(q_{n}\right)\right|}{\sum_{k \geq 1}\left|\widehat{f}\left(k q_{n}\right)\right|}>c_{0}>0, \\
\frac{\left|\widehat{f}\left(q_{n}\right)\right|}{\left|\widehat{f}\left(q_{n}\right)\right|+\sum_{k \geq 1}\left|\widehat{f}\left(k \frac{r}{s} q_{n}\right)\right|}>c_{0}>0, \text { whenever } s \mid q_{n},
\end{gathered}
$$

\footnotetext{
12 In [18] $f$ is assumed to be of class $C^{2}$ and the proof, in fact, requires that $\sum n|\widehat{f}(n)|<\infty$. See [21] for the proof of Theorem 2.5.1 with $f \in C^{1+\delta}(\mathbb{T})$ for some $\delta>0$.
} 
and

$$
\frac{\left|\alpha-\frac{p_{n}}{q_{n}}\right| q_{n}}{\left|\widehat{f}\left(q_{n}\right)\right|} \rightarrow 0
$$

Then for each $\lambda \in \mathbb{S}^{1}, h \in \mathbb{R}$ and $A, B \in \mathbb{R}$ with $A^{2}+B^{2} \neq 0$ the cocycle $\lambda e^{2 \pi i\left(A f^{(r)}(r x)+B f^{(s)}(s x+h)\right)}$ is not a $T$-coboundary.

Proof Fix $A, B \in \mathbb{R}$ with $A^{2}+B^{2} \neq 0$, relatively prime numbers $r, s$ and $h \in \mathbb{R}$. Set

$$
F(x):=A f^{(r)}(r x)+B f^{(s)}(s x+h) .
$$

For any $k \in \mathbb{N}$ we have

$$
f^{(k)}(x)=\sum_{n \in \mathbb{Z}} \widehat{f}(n) \frac{1-e^{2 \pi i n k \alpha}}{1-e^{2 \pi i n \alpha}} e^{2 \pi i n x}
$$

Therefore,

$$
F(x)=A \sum_{n \in \mathbb{Z}} \widehat{f}(n) \frac{1-e^{2 \pi i n r \alpha}}{1-e^{2 \pi i n \alpha}} e^{2 \pi i n r x}+B \sum_{n \in \mathbb{Z}} \widehat{f}(n) \frac{1-e^{2 \pi i n s \alpha}}{1-e^{2 \pi i n \alpha}} e^{2 \pi i n(s x+h)}
$$

Suppose first that $A \cdot B=0$. We may assume without loss of generality that $A=0$ and $B \neq 0$. Then, for all $n \in \mathbb{N}$, by (29), we have

$$
\widehat{F}(s n)=B \widehat{f}(n) \frac{1-e^{2 \pi i n s \alpha}}{1-e^{2 \pi i n \alpha}} e^{2 \pi i n h} .
$$

Therefore, since

$$
\begin{gathered}
\left|\frac{1-e^{2 \pi i q_{n} s \alpha}}{1-e^{2 \pi i q_{n} \alpha}}\right| \rightarrow s \text { as } n \rightarrow \infty, \\
\left|\widehat{F}\left(s q_{n}\right)\right| \geq|B| \cdot \frac{s}{2} \cdot\left|\widehat{f}\left(q_{n}\right)\right| \text { for } n \text { sufficiently large }
\end{gathered}
$$

and

$$
\left|\widehat{F}\left(m s q_{n}\right)\right|=|B| \cdot\left|\widehat{f}\left(m q_{n}\right)\right| \cdot\left|\frac{1-e^{2 \pi i m s q_{n} \alpha}}{1-e^{2 \pi i m q_{n} \alpha}}\right| \leq|B| \cdot s \cdot\left|\widehat{f}\left(m q_{n}\right)\right|
$$

for all $n \in \mathbb{N}, m \in \mathbb{Z}$. It follows immediately by (31) and (32) that for $n$ sufficiently large

$$
\begin{gathered}
\frac{\left|\widehat{F}\left(s q_{n}\right)\right|}{\sum_{m \geq 1}\left|\widehat{F}\left(m s q_{n}\right)\right|} \geq \frac{|B| \cdot \frac{s}{2} \cdot\left|\widehat{f}\left(q_{n}\right)\right|}{\sum_{m \geq 1}|B| \cdot s \cdot\left|\widehat{f}\left(m q_{n}\right)\right|}=\frac{\left|\widehat{f}\left(q_{n}\right)\right|}{2 \sum_{m \geq 1}\left|\widehat{f}\left(m q_{n}\right)\right|} \\
\frac{\left|\alpha-\frac{s p_{n}}{s q_{n}}\right| s q_{n}}{\left|\widehat{F}\left(s q_{n}\right)\right|} \leq \frac{2}{|B|} \cdot \frac{\left|\alpha-\frac{p_{n}}{q_{n}}\right| q_{n}}{\left|\widehat{f}\left(q_{n}\right)\right|} .
\end{gathered}
$$


Now, (33) and (34) and the assumptions (26) and (28) imply that for $n$ sufficiently large

$$
\frac{\left|\widehat{F}\left(s q_{n}\right)\right|}{\sum_{m \geq 1}\left|\widehat{F}\left(m s q_{n}\right)\right|}>\frac{c_{0}}{2}>0 \quad \text { and } \quad \frac{\left|\alpha-\frac{s p_{n}}{s q_{n}}\right| s q_{n}}{\left|\widehat{F}\left(s q_{n}\right)\right|} \rightarrow 0
$$

In view of Theorem 2.5.1, this completes the proof in case $A \cdot B=0$.

Suppose now that $A \cdot B \neq 0$. Applying the fact that for any absolutely summable sequence $\left(y_{n}\right)_{n \in \mathbb{Z}}$

$$
\sum_{n \in \mathbb{Z}} y_{n}=\sum_{r \mid n} y_{n}+\sum_{r \nmid n} y_{n}=\sum_{n \in \mathbb{Z}} y_{n r}+\sum_{r \nmid n} y_{n}=\sum_{s \mid n} y_{n} \frac{r}{s}+\sum_{r \nmid n} y_{n}
$$

13 to the second summand in formula (29), we obtain

$$
\begin{aligned}
& F(x)=\sum_{s \mid n} \underbrace{\left(A \widehat{f}(n) \frac{1-e^{2 \pi i n r \alpha}}{1-e^{2 \pi i n \alpha}}+B \widehat{f}\left(n \frac{r}{s}\right) \frac{1-e^{2 \pi i n r \alpha}}{1-e^{2 \pi i n \frac{r}{s} \alpha}} e^{2 \pi i n \frac{r}{s} h}\right)}_{a_{r n}} e^{2 \pi i n r x} \\
& +A \sum_{s \nmid n} \widehat{f}(n) \frac{1-e^{2 \pi i r n \alpha}}{1-e^{2 \pi i n \alpha}} e^{2 \pi i n r x}+B \sum_{r \nmid n} \widehat{f}(n) \frac{1-e^{2 \pi i n s \alpha}}{1-e^{2 \pi i n \alpha}} e^{2 \pi i n h} e^{2 \pi i n s x} .
\end{aligned}
$$

For $m \in \mathbb{N}$, let $B_{m}:=\left\{n \in \mathbb{N}: m \nmid q_{n}\right\}$. We will consider the following cases:

1. $B_{r}$ or $B_{s}$ is infinite,

2. Both sets $B_{r}$ and $B_{s}$ are finite.

We will cover first case (1). Without loss of generality we may assume that $B_{r}$ is infinite. Suppose that

$$
\lambda e^{2 \pi i\left(A f^{(r)}(r x)+B f^{(s)}(s x+h)\right)} \text { is a coboundary. }
$$

Since $f^{(r)}(r x)$ is $\frac{1}{r}$-periodic, it follows immediately that

$$
\lambda e^{2 \pi i\left(A f^{(r)}(r x)+B f^{(s)}\left(s x+\frac{s}{r}+h\right)\right)} \text { is also a coboundary. }
$$

Hence, dividing the expression from (36) by the one from (37), we conclude that

$$
e^{2 \pi i B\left(f^{(s)}(s x)-f^{(s)}\left(s x+\frac{s}{r}\right)\right)} \text { is a coboundary as well. }
$$

\footnotetext{
13 These equalities hold for all $r, s \in \mathbb{N}$.
} 
We will show that this is impossible in view of Theorem 2.5.1. Indeed, we have

$$
g(x):=f^{(s)}(s x)-f^{(s)}\left(s x+\frac{s}{r}\right)=\sum_{n \in \mathbb{Z}} \widehat{f}(n) \frac{1-e^{2 \pi i n s \alpha}}{1-e^{2 \pi i n \alpha}}\left(1-e^{2 \pi i n \frac{s}{r}}\right) e^{2 \pi i n s x} .
$$

We claim that for $n \in B_{r}$,

$$
\frac{\left|\widehat{g}\left(s q_{n}\right)\right|}{\sum_{k \geq 1}\left|\widehat{g}\left(k s q_{n}\right)\right|}>c_{1} \quad \text { for some } c_{1}>0
$$

and

$$
\frac{\left|\alpha-\frac{s p_{n}}{s q_{n}}\right| s q_{n}}{\left|\widehat{g}\left(s q_{n}\right)\right|} \rightarrow 0
$$

Indeed, for $n \in B_{r}$, we have

$$
\left|\widehat{g}\left(s q_{n}\right)\right|=\left|\widehat{f}\left(q_{n}\right)\right| \cdot\left|\frac{1-e^{2 \pi i s q_{n} \alpha}}{1-e^{2 \pi i q_{n} \alpha}}\right| \cdot\left|e^{2 \pi i q_{n} \frac{s}{r}}-1\right| .
$$

Since $r \nmid q_{n}$ and $r$ and $s$ are relatively prime,

$$
\left|e^{2 \pi i q_{n} \frac{s}{r}}-1\right| \geq\left|e^{2 \pi i \frac{1}{r}}-1\right|=: c_{2}>0 .
$$

Using again (30), we obtain from (41) and (42) that for $n$ sufficiently large,

$$
\left|\widehat{g}\left(s q_{n}\right)\right| \geq \frac{c_{2} s}{2} \cdot\left|\widehat{f}\left(q_{n}\right)\right|
$$

On the other hand, since $\left|\frac{1-e^{2 \pi i k s q_{n} \alpha}}{1-e^{2 \pi i k q_{n} \alpha}}\right| \leq s$,

$$
\begin{aligned}
\sum_{k \geq 1}\left|\widehat{g}\left(k s q_{n}\right)\right| & =\sum_{k \geq 1}\left|\widehat{f}\left(k q_{n}\right)\right| \cdot\left|\frac{1-e^{2 \pi i k s q_{n} \alpha}}{1-e^{2 \pi i k q_{n} \alpha}}\right| \cdot\left|e^{2 \pi i q_{n} \frac{s}{r}}-1\right| \\
& \leq \sum_{k \geq 1}\left|\widehat{f}\left(k q_{n}\right)\right| \cdot s \cdot\left|e^{2 \pi i q_{n} \frac{s}{r}}-1\right| \leq 2 s \sum_{k \geq 1}\left|\widehat{f}\left(k q_{n}\right)\right|
\end{aligned}
$$

Thus, using (43), (44) and (26) we obtain, for $n$ large enough,

$$
\frac{\left|\widehat{g}\left(s q_{n}\right)\right|}{\sum_{k \geq 1}\left|\widehat{g}\left(k s q_{n}\right)\right|} \geq \frac{c_{2} s\left|\widehat{f}\left(q_{n}\right)\right|}{4 s \sum_{k \geq 1}\left|\widehat{f}\left(k q_{n}\right)\right|}>\frac{c_{0} c_{2}}{4},
$$

which shows that (39) holds. Using (43) and (28), we obtain that (40) also holds:

$$
\frac{\left|\alpha-\frac{s p_{n}}{s q_{n}}\right| s q_{n}}{\left|\widehat{g}\left(s q_{n}\right)\right|} \leq \frac{2\left|\alpha-\frac{p_{n}}{q_{n}}\right| s q_{n}}{c_{2} s\left|\widehat{f}\left(q_{n}\right)\right|}=\frac{2}{c_{2}} \cdot \frac{\left|\alpha-\frac{p_{n}}{q_{n}}\right| q_{n}}{\left|\widehat{f}\left(q_{n}\right)\right|} \rightarrow 0 .
$$


It follows from Theorem 2.5.1 that $\lambda e^{2 \pi i B\left(f^{(s)}(s x)-f^{(s)}\left(s x+\frac{s}{r}\right)\right)}$ cannot be a coboundary, contrary to (38). This completes the proof in case (1).

We cover now case (2). We claim that for $n \notin B_{s}$ (i.e. $n$ such that $s \mid q_{n}$ ), for some $c_{3}>0$, we have

$$
\frac{\left|a_{r q_{n}}\right|}{\sum_{m \geq 1}\left|a_{m r q_{n}}\right|}>c_{3}>0
$$

and

$$
\frac{\left|\alpha-\frac{r p_{n}}{r q_{n}}\right| r q_{n}}{\left|a_{r q_{n}}\right|} \rightarrow 0 .
$$

To prove (45), we will estimate $\left|a_{r q_{n}}\right|$ from below and $\sum_{m \geq 1}\left|a_{m r q_{n}}\right|$ from above in an appropriate way. We begin by estimating $\left|a_{r q_{n}}\right|$. We have

$$
\begin{aligned}
\left|a_{r q_{n}}\right| & \geq|A| \cdot\left|\widehat{f}\left(q_{n}\right)\right| \cdot\left|\frac{1-e^{2 \pi i r q_{n} \alpha}}{1-e^{2 \pi i q_{n} \alpha}}\right|-|B| \cdot\left|\widehat{f}\left(q_{n} \frac{r}{s}\right)\right| \cdot\left|\frac{1-e^{2 \pi i q_{n} r \alpha}}{1-e^{2 \pi i q_{n} \frac{r}{s}} \alpha}\right| \\
& \geq|A| \cdot\left|\widehat{f}\left(q_{n}\right)\right| \cdot \frac{r}{2}-|B| \cdot\left|\widehat{f}\left(q_{n} \frac{r}{s}\right)\right| \cdot\left|\frac{1-e^{2 \pi i q_{n} r \alpha}}{1-e^{2 \pi i q_{n} \frac{r}{s} \alpha}}\right|,
\end{aligned}
$$

where the latter inequality follows from (30) and is valid for $n$ sufficiently large. It follows by (27) that for $n \notin B_{S}$,

$$
\left|\widehat{f}\left(q_{n} \frac{r}{s}\right)\right|<\frac{1}{c_{0}}\left|\widehat{f}\left(q_{n}\right)\right| .
$$

We will now estimate $\left|1-e^{2 \pi i q_{n} \frac{r}{s} \alpha}\right|$. Notice that

$$
\frac{2}{\pi}<\frac{\left|e^{2 \pi i x}-e^{2 \pi i y}\right|}{|x-y|}<1
$$

for $x, y \in \mathbb{R}$ such that $0<|x-y|<1$. Since $\left|1-e^{2 \pi i q_{n} \alpha}\right| \rightarrow 0$, for $n$ sufficiently large,

$$
\left|1-e^{2 \pi i q_{n} \alpha}\right|<\frac{s}{\pi r}\left|1-e^{2 \pi i \frac{1}{s}}\right| .
$$

Therefore and by (49), for such $n$, we have

$$
\begin{aligned}
\left|e^{2 \pi i \frac{\left[q_{n} \alpha\right]}{s}}-e^{2 \pi i q_{n} \frac{1}{s} \alpha}\right| & <\left|\frac{\left[q_{n} \alpha\right]}{s}-q_{n} \frac{1}{s} \alpha\right|=\frac{1}{s} \cdot\left|\left[q_{n} \alpha\right]-q_{n} \alpha\right| \\
& <\frac{\pi}{2 s} \cdot\left|e^{2 \pi i\left[q_{n} \alpha\right]}-e^{2 \pi i q_{n} \alpha}\right|=\frac{\pi}{2 s} \cdot\left|1-e^{2 \pi i q_{n} \alpha}\right| \\
& <\frac{\pi}{2 s} \cdot \frac{s}{\pi r} \cdot\left|1-e^{2 \pi i \frac{1}{s}}\right|=\frac{1}{2 r} \cdot\left|1-e^{2 \pi i \frac{1}{s}}\right| .
\end{aligned}
$$

Since for all $x, y \in \mathbb{R}$ and all $k \in \mathbb{N}$

$$
\left|e^{2 \pi i k x}-e^{2 \pi i k y}\right| \leq k\left|e^{2 \pi i x}-e^{2 \pi i y}\right|,
$$


it follows from (50) that

$$
\left|e^{2 \pi i \frac{\left[q_{n} \alpha\right] r}{s}}-e^{2 \pi i q_{n} \frac{r}{s}} \alpha\right|<\frac{1}{2}\left|1-e^{2 \pi i \frac{1}{s}}\right|
$$

Since $q_{n}$ is a denominator of $\alpha$ and $s \mid q_{n}$, we have $\left|c-\frac{q_{n}}{s} \alpha\right|>\left|p_{n}-q_{n} \alpha\right|$ for all $0 \leq c \leq \frac{q_{n}}{s}$ (see (56) in Sect. 1). Using this inequality and (49), we obtain

$$
\frac{\pi}{2}\left|1-e^{2 \pi i \frac{q_{n}}{s} \alpha}\right|>\left|c-\frac{q_{n}}{s} \alpha\right|>\left|p_{n}-q_{n} \alpha\right|>\left|1-e^{2 \pi i q_{n} \alpha}\right| .
$$

By the first two lines of (50) and by (52), we have

$$
\left|e^{2 \pi i \frac{\left[q_{n} \alpha\right]}{s}}-e^{2 \pi i q_{n} \frac{1}{s} \alpha}\right|<\frac{\pi}{2 s}\left|1-e^{2 \pi i q_{n} \alpha}\right|<\frac{\pi}{2 s} \cdot \frac{\pi}{2}\left|1-e^{2 \pi i \frac{q_{n}}{s} \alpha}\right| .
$$

Suppose that $s \mid\left[q_{n} \alpha\right]$. Then (53) implies $1<\frac{\pi}{2 s} \cdot \frac{\pi}{2}$, i.e. $s<(\pi / 2)^{2}$. This is however impossible, as $s \geq 3$. Therefore $s \nmid\left[q_{n} \alpha\right]$, which implies $s \nmid\left[q_{n} \alpha\right] r$, whence

$$
\left|1-e^{2 \pi i \frac{\left[q_{n} \alpha\right] r}{s}}\right| \geq\left|1-e^{2 \pi i \frac{1}{s}}\right|
$$

Therefore, by (51) and (54),

$$
\begin{aligned}
\left|1-e^{2 \pi i q_{n} \frac{r}{s} \alpha}\right| & \geq\left|1-e^{2 \pi i \frac{\left[q_{n} \alpha\right] r}{s}}\right|-\left|e^{2 \pi i \frac{\left[q_{n} \alpha\right] r}{s}}-e^{2 \pi i q_{n} \frac{r}{s} \alpha}\right| \\
& \geq\left|1-e^{2 \pi i \frac{\left[q_{n} \alpha\right] r}{s}}\right|-\frac{1}{2}\left|1-e^{2 \pi i \frac{1}{s}}\right| \geq \frac{1}{2}\left|1-e^{2 \pi i \frac{1}{s}}\right|=: c_{4}>0 .
\end{aligned}
$$

It follows that

$$
\left|\frac{1-e^{2 \pi i r q_{n} \alpha}}{1-e^{2 \pi i q_{n} \frac{r}{s} \alpha}}\right| \leq \frac{1}{c_{4}}\left|1-e^{2 \pi i r q_{n} \alpha}\right| \leq \frac{r}{c_{4}}\left|1-e^{2 \pi i q_{n} \alpha}\right| \rightarrow 0 .
$$

This and (47), (48) imply that for $n$ sufficiently large

$$
\left|a_{r q_{n}}\right| \geq c_{5}\left|\widehat{f}\left(q_{n}\right)\right| \text { for some } c_{5}>0 .
$$

We will estimate now $\sum_{m \geq 1}\left|a_{m r q_{n}}\right|$. By (26) and (27) we have

$$
\sum_{m \geq 1}\left|\widehat{f}\left(m q_{n}\right)\right|<\frac{1}{c_{0}}\left|\widehat{f}\left(q_{n}\right)\right| \text { and } \sum_{m \geq 1}\left|\widehat{f}\left(m q_{n} \frac{r}{s}\right)\right|<\frac{1}{c_{0}}\left|\widehat{f}\left(q_{n}\right)\right| .
$$

Hence

$$
\sum_{m \geq 1}\left|a_{m r q_{n}}\right| \leq \frac{|A| r+|B| s}{c_{0}} \cdot\left|\widehat{f}\left(q_{n}\right)\right|
$$


Using this estimate and (55), we obtain

$$
\frac{\left|a_{r q_{n}}\right|}{\sum_{m \geq 1} \mid a_{m r q_{n} \mid}} \geq \frac{c_{5}\left|\widehat{f}\left(q_{n}\right)\right|}{\frac{|A| r+|B| s}{c_{0}}\left|\widehat{f}\left(q_{n}\right)\right|}=\frac{c_{0} c_{5}}{|A| r+|B| s}>0
$$

and (45) follows. Notice that (55) together with (28) implies that also (46) is true. By Theorem 2.5.1, we conclude that $\lambda e^{2 \pi i F(x)}$ cannot be a coboundary, which completes the proof in case (2).

Remark 2.5.3 Recall that if $f \in C^{1+\delta}(\mathbb{T})$ for some $\delta>0$ then $\widehat{f}(n)=\mathrm{o}\left(1 / n^{1+\delta^{\prime}}\right)$ for $0<\delta^{\prime}<\delta$ (the speed of convergence to zero depends on $\delta^{\prime}$ ).

Lemma 2.5.4 (cf. Lemma 4 in [21]) Let $g: \mathbb{N} \rightarrow(0, \infty)$ be a non-increasing positive function such that $g(m n) \leq g(m) g(n)$ for all $m, n \in \mathbb{N}$ and $\sum_{m \geq 1} g(m)<\infty$. Let $\left(x_{n}\right)_{n \in \mathbb{N}} \subset[0, \infty)$ be a summable sequence such that $x_{n}=o(g(n)), n \in \mathbb{N}$. Let $\left(x_{n_{k}}\right)_{k \in \mathbb{N}}$ be a subsequence of $\left(x_{n}\right)_{n \in \mathbb{N}}$ such that $x_{n_{k}}>0$. Let $b \geq 1$ and let

$$
\varepsilon_{k}=\frac{x_{n_{k}}}{x_{n_{k}}+\sum_{m \geq 1} x_{m\left[b n_{k}\right]}} .
$$

Then $\varepsilon_{k} \not \nrightarrow 0$.

Proof We will choose a subsequence of $\left(\varepsilon_{k}\right)_{k \in \mathbb{N}}$ recursively. Let $C:=\sum_{m \geq 1} g(m)$. Let $k_{1} \geq 1$ and $\delta_{1}>0$ be such that

$$
\frac{x_{n_{k_{1}}}}{g\left(n_{k_{1}}\right)}>\delta_{1} \text { and } \frac{x_{n}}{g(n)} \leq \delta_{1} \quad \text { for } n>n_{k_{1}}
$$

Suppose first that $\left[b n_{k_{1}}\right]=n_{k_{1}}$. Then

$$
\begin{aligned}
& x_{n_{k_{1}}}+\sum_{m \geq 1} x_{m\left[b n_{k_{1}}\right]}=2 x_{n_{k_{1}}}+\sum_{m \geq 2} x_{m\left[b n_{k_{1}}\right]} \leq 2 x_{n_{k_{1}}}+\sum_{m \geq 2} g\left(m\left[b n_{k_{1}}\right]\right) \delta_{1} \\
& \leq 2 x_{n_{k_{1}}}+g\left(\left[b n_{k_{1}}\right]\right) \delta_{1} \sum_{m \geq 2} g(m) \leq 2 x_{n_{k_{1}}}+g\left(n_{k_{1}}\right) \delta_{1} C<(C+2) x_{n_{k_{1}}} .
\end{aligned}
$$

Suppose now that $\left[b n_{k_{1}}\right]>n_{k_{1}}$. In a similar way as before, we obtain

$$
x_{n_{k_{1}}}+\sum_{m \geq 1} x_{m\left[b n_{k_{1}}\right]} \leq x_{n_{k_{1}}}+g\left(n_{k_{1}}\right) \delta_{1} \sum_{m \geq 1} g(m)<(C+1) x_{n_{k_{1}}} \text {. }
$$

Once we have chosen $k_{1}, \ldots, k_{j}$, we pick $k_{j+1}>k_{j}$ and $\delta_{j+1}>0$ such that

$$
\frac{x_{n_{k_{j+1}}}}{g\left(n_{k_{j+1}}\right)}>\delta_{j+1} \text { and } \frac{x_{n}}{g(n)} \leq \delta_{j+1} \quad \text { for } n>n_{k_{j+1}} \text {. }
$$


As before, we obtain

$$
\sum_{m \geq 1} x_{m\left[b n_{k_{j+1}}\right]}<(C+2) x_{n_{k_{j+1}}}
$$

which completes the proof.

Remark 2.5.5 Given $b_{1}, \ldots, b_{l} \geq 1$, under the assumptions of the above lemma, by a diagonalizing procedure we can find an increasing sequence $\left(n_{k}\right)_{k \in \mathbb{N}} \subset \mathbb{N}$ such that

$$
\varepsilon_{n_{k}}\left(b_{i}\right)>c>0 \text { for } 1 \leq i \leq l \text {. }
$$

Corollary 2.5.6 Suppose that $f \in C^{1+\delta}(\mathbb{T})$ for some $\delta>0$ and $f$ is not a trigonometric polynomial. Then for a generic $\alpha$ and any $A, B \in \mathbb{R}$ with $A^{2}+B^{2} \neq 0$, any relatively prime numbers $r, s$, any $h \in \mathbb{R}$ and any $\lambda \in \mathbb{T}$, the cocycle $\lambda e^{2 \pi i\left(A f^{(r)}(r x)+B f^{(s)}(s x+h)\right)}$ is not a $T$-coboundary for $T x=x+\alpha$.

Proof Notice first that if

$$
\lambda e^{2 \pi i\left(A f^{(r)}(r x)+B f^{(s)}(s x+h)\right)} \text { is a } T \text {-coboundary for } T x=x+\alpha
$$

then also

$$
\lambda e^{2 \pi i\left(A f^{(r)}\left(r x-\frac{h r}{s}\right)+B f^{(s)}(s x)\right)} \text { is a } T \text {-coboundary for } T x=x+\alpha .
$$

Therefore, we may assume without loss of generality that $r>s$. Let $\left(q_{n}\right)_{n \in \mathbb{N}}$ be such that $\widehat{f}\left(q_{n}\right) \neq 0$. Then by (57), for a residual set of irrationals $\alpha$, we have

$$
\frac{\left|\alpha-\frac{p_{n}}{q_{n}}\right| q_{n}}{\left|\widehat{f}\left(q_{n}\right)\right|} \rightarrow 0
$$

along some subsequence of $\left(q_{n}\right)_{n \in \mathbb{N}}$ which, for convenience, we will still denote by $\left(q_{n}\right)_{n \in \mathbb{N}}$. In other words, (28) holds. It follows from Remark 2.5.3, Lemma 2.5.4 and Remark 2.5.5 (for $l=2, b_{1}=1, b_{2}=\frac{r}{s}$ ) that (26) and (27) also hold. Therefore, we can apply Theorem 2.5.2 to complete the proof.

Remark 2.5.7 Let $c \neq 0, r, s, A, B \in \mathbb{Z}$ be such that $A r^{2}+B s^{2} \neq 0$. Let $\varphi(x)=$ $c x+f(x)$, where $f: \mathbb{R} \rightarrow \mathbb{R}$ is of class $C^{1+\delta}$ for some $\delta>0$ and periodic of period 1. Since the topological degree of $e^{2 \pi i\left(A \varphi^{(r)}(r \cdot)+B \varphi^{(s)}(s \cdot+h)\right)}$ is equal to $\left(A r^{2}+B s^{2}\right) c$, an immediate consequence of (60) is that the cocycle $e^{2 \pi i\left(A \varphi^{(r)}(r \cdot)+B \varphi^{(s)}(s \cdot+h)\right)}$ is not a coboundary for all $h \in \mathbb{R}$. On the other hand, if $A r^{2}+B s^{2}=0$, then for some $\lambda$ of modulus 1 , we have

$$
e^{2 \pi i\left(A \varphi^{(r)}(r \cdot)+B \varphi^{(s)}(s \cdot+h)\right)}=\lambda \cdot e^{2 \pi i\left(A f^{(r)}(r \cdot)+B f^{(s)}(s \cdot+h)\right)} .
$$


Corollary 2.5.8 Let $c \in \mathbb{Z}$ and suppose that $f \in C^{1+\delta}(\mathbb{T})$ for some $\delta>0$ and $f$ is not a trigonometric polynomial. Let $\varphi(x)=c x+f(x)$. Then for a generic $\alpha$ the cocycle $\left(e^{2 \pi i \varphi^{(r)}(r)}, e^{2 \pi i \varphi^{(s)}(s \cdot+h)}\right)$ is ergodic (as a cocycle over $T x=x+\alpha$ ) for all relatively prime numbers $r \neq s$ and any $h \in \mathbb{R}$.

Proof In view of Remark 2.5.10, the assertion follows immediately from Corollary 2.5.6 and Remark 2.5.7.

Proof of Theorem 1.0.3 Recall that $T_{c, f}(x, y)=(x+\alpha, y+c x+f(x))$. We can assume that $f$ is not a trigonometric polynomial. Indeed, otherwise $f$ is a coboundary with the transfer function also being a trigonometric polynomial and the problem is reduced to the affine case.

It follows from Lemma 2.2.2 and Corollary 2.5.8 that for a generic $\alpha$ the decomposition of $\mathbb{T}^{4}$ into minimal components of $\left(T_{c, f}\right)^{r} \times\left(T_{c, f}\right)^{s}$ is the same as the decomposition into ergodic components: it consists of sets $I_{c_{1}}, c_{1} \in\left[0, \frac{1}{r}\right.$ ) (see (9) on page 9$)$. Moreover, each ergodic component is uniquely ergodic. Therefore, all points are generic for $\left(T_{c, f}\right)^{r} \times\left(T_{c, f}\right)^{s}$ (for the relevant invariant measures). Thus, conditions (a) and (b) are satisfied.

Finally, notice that by Lemma (2.4.8), $\mathcal{F}:=\widehat{\mathbb{T}}^{2}$ satisfies condition (c). This completes the proof.

Open Access This article is distributed under the terms of the Creative Commons Attribution 4.0 International License (http://creativecommons.org/licenses/by/4.0/), which permits unrestricted use, distribution, and reproduction in any medium, provided you give appropriate credit to the original author(s) and the source, provide a link to the Creative Commons license, and indicate if changes were made.

\section{Appendix}

\section{Notation and basic facts}

Automorphisms of standard Borel spaces Let $(X, \mathcal{B}, \mu)$ be a standard probability Borel space. By $\operatorname{Aut}(X, \mathcal{B}, \mu)$ we denote the space of all bi-measurable measure-preserving bijections of $X$ which we call automorphisms.

Irrational rotation We identify the multiplicative circle $\mathbb{S}^{1}=\{z \in \mathbb{C}:|z|=1\}$ and $\mathbb{T}=\mathbb{R} / \mathbb{Z}$ with $X=[0,1)$ with addition $\bmod 1$. Therefore, real functions defined on the circle are identified with one-periodic functions defined on $\mathbb{R}$. Let $\lambda_{\mathbb{T}}$ denote Lebesgue measure on $X$.

Assume that $T: X \rightarrow X$ is an irrational rotation, $T x=x+\alpha(\bmod 1), x \in X$. Clearly $T \in \operatorname{Aut}\left(X, \mathcal{B}(X), \lambda_{\mathbb{T}}\right)$. Let $\alpha=\left[0 ; a_{1}, a_{2}, \ldots\right]$ be the continued fraction expansion of $\alpha$. Let

$$
\begin{aligned}
& q_{0}=1, q_{1}=a_{1}, q_{n+1}=a_{n+1} q_{n}+q_{n-1}, \\
& p_{0}=0, p_{1}=1, p_{n+1}=a_{n+1} p_{n}+p_{n-1}
\end{aligned}
$$

for $n \geq 1$. The rationals $p_{n} / q_{n}$ are called the convergents of $\alpha$. 
Recall (see e.g. [20]) that every convergent $p_{n} / q_{n}$ is a best approximation of $\alpha$ in the following sense:

$$
\text { if } \frac{c}{d} \neq \frac{p_{n}}{q_{n}} \text { and } 0<d \leq q_{n} \text { then }|c-d \alpha|>\left|p_{n}-q_{n} \alpha\right| .
$$

Recall also (see e.g. [21]) that given an infinite set $\left\{q_{n}: n \in \mathbb{N}\right\} \subset \mathbb{N}$ and a positive real valued function $R=R\left(q_{n}\right)$, the set

$$
\begin{aligned}
\mathcal{A}= & \left\{\alpha \in[0,1): \text { for infinitely many } n \text { we have }\left|\alpha-\frac{p_{n}}{q_{n}}\right|<R\left(q_{n}\right),\right. \\
& \text { where } \left.\frac{p_{n}}{q_{n}} \text { are convergents of } \alpha\right\} \text { is residual in } \mathbb{T} .
\end{aligned}
$$

Cocycles and group extensions Let $T \in \operatorname{Aut}(X, \mathcal{B}, \mu)$. For a locally compact second countable Abelian group $G^{14}$ with a Haar measure $\lambda_{G}$, a measurable map $\varphi: \mathbb{Z} \times X \rightarrow$ $G$ is called a cocycle if

$$
\varphi^{(n+m)}(x)=\varphi^{(n)}(x) \varphi^{(m)}\left(T^{n} x\right) \text { for each } n, m \in \mathbb{Z} .
$$

The generator $\varphi(x)=\varphi^{(1)}(x)$ determines $\varphi^{(n)}(x)$ for any $(n, x) \in \mathbb{Z} \times X$ :

$$
\varphi^{(n)}(x)= \begin{cases}\varphi(x) \cdot \varphi(T x) \cdot \ldots \cdot \varphi\left(T^{n-1} x\right), & \text { if } n>0 \\ 1, & \text { if } n=0 \\ \left(\varphi\left(T^{n} x\right) \cdot \ldots \cdot \varphi\left(T^{-1} x\right)\right)^{-1}, & \text { if } n<0\end{cases}
$$

Thus, we call a cocycle any measurable function $\varphi: X \rightarrow G$ as well. Given $\varphi$, consider a $G$-extension of $T$, acting on $\left(X \times G, \mu \otimes \lambda_{G}\right)$, defined by the formula

$$
T_{\varphi}(x, g)=(T x, \varphi(x) g) .
$$

A cocycle $\varphi$ is called a $T$-coboundary (or simply a coboundary) if it is of the form

$$
\varphi(x)=\xi(x)(\xi(T x))^{-1}
$$

for some measurable function $\xi: X \rightarrow G$ (called a transfer function). Two cocycles $\phi, \psi: X \rightarrow G$ are cohomologous if for some measurable function $f: X \rightarrow G$ we have

$$
(f(T x))^{-1} \phi(x) f(x)=\psi(x) .
$$

Analogous notions to the above ones are also present in topological dynamics. Let $T: X \rightarrow X$ be a minimal homeomorphism of a compact metric space. Let $\varphi: X \rightarrow G$ be a continuous function. We say that $\varphi$ is a topological $T$-coboundary if it is a measurable $T$-coboundary with a continuous transfer function.

14 We use multiplicative notation in $G$. 


\section{Compact group extensions: ergodicity and minimality}

Let $G$ be a compact Abelian metrizable group and assume that $T \in \operatorname{Aut}(X, \mathcal{B}, \mu)$ is ergodic. Let $\varphi: X \rightarrow G$ be a cocycle. Then

$T_{\varphi}$ is ergodic if and only if the trivial solution $\chi=\mathbb{1}, \xi=$ $\mathrm{m}$ const is the only solution of $\chi \circ \varphi=\xi / \xi \circ T$ in $\chi \in \widehat{G}$ and measurable $\xi: X \rightarrow \mathbb{S}^{1}$.

In particular (see [11]),

If $T x=x+\alpha, \varphi: \mathbb{T} \rightarrow \mathbb{S}^{1}$ is Lipschitz with non-zero degree then $T_{\varphi}$ is ergodic.

If $T_{\varphi}$ is ergodic, we will also say that $\varphi$ is ergodic (for $T$ ).

Recall that $G$ acts on $X \times G$ via $g \mapsto R_{g}$, where:

$$
R_{g}(x, h):=\left(x, h g^{-1}\right) \quad \text { for }(x, h) \in X \times G, g \in G
$$

Ergodic components Let $\varphi: X \rightarrow G$ be a cocycle. Let $\mathcal{P}\left(T_{\varphi}, \mathcal{B}, \mu\right)$ stand for the set of $T_{\varphi}$-invariant Borel measures whose projection on $X$ is $\mu$. Fix an ergodic measure $\lambda \in \mathcal{P}\left(T_{\varphi}, \mathcal{B}, \mu\right)$. Denote by $H$ be the stabilizer of $\lambda$ in $G$, i.e.

$$
H:=\left\{g \in G: \lambda \circ R_{g}=\lambda\right\}
$$

Notice that

$$
\Gamma:=\{\chi \in \widehat{G}: \chi \circ \varphi \text { is a coboundary }\}
$$

is a (closed) subgroup of $\widehat{G}$ and let

$$
F=\text { ann } \Gamma:=\{g \in G: \text { for each } \chi \in \Gamma, \chi(g)=1\} .
$$

Proposition 2.5 .9 (see e.g. [22]) The system $\left(X \times G, T_{\varphi}, \lambda\right)$ is isomorphic to $(X \times$ $\left.H, T_{\psi}, \mu \otimes \lambda_{H}\right)$ for some ergodic $\psi: X \rightarrow H$. Moreover, $F=H$.

Remark 2.5.10 In view of (59), $T_{\varphi}$ is ergodic if and only if $\Gamma=\{\mathbb{1}\}$.

Minimal components Let $T: X \rightarrow X$ be a minimal homeomorphism of a compact metric space. Let $\varphi: X \rightarrow G$ be a continuous function and let $M$ be a minimal component of $T_{\varphi}$, i.e. $M \subset X \times G$ is closed and invariant with no proper subsets having the same properties. Let $H_{t o p}$ be the stabilizer of $M$ in $G$, i.e.

$$
H_{\text {top }}:=\left\{g \in G: R_{g}(M)=M\right\}
$$


(this definition is independent of the initial choice of $M$ ). Notice that

$$
\Gamma_{\text {top }}:=\{\chi \in \widehat{G}: \chi \circ \varphi \text { is a topological coboundary }\}
$$

is a closed subgroup of $\widehat{G}$ and let

$$
F_{\text {top }}:=\operatorname{ann} \Gamma_{\text {top }}=\left\{g \in G: \text { for each } \chi \in \Gamma_{t o p}, \chi(g)=1\right\} .
$$

Lemma 2.5.11 ([27]) There exists a continuous map $\tau: X \rightarrow G / H_{\text {top }}$ such that

$$
\tau(T x)=\varphi(x) \tau(x)
$$

Moreover, $M:=\cup_{x \in X}\{x\} \times \tau(x)$ is a minimal set.

The proof of the following result is analogous to the one in the measure-theoretical case. We include it here for the sake of completeness.

Proposition 2.5.12 $H_{t o p}=F_{t o p}$.

Proof Let $g_{0} \in H_{\text {top }}$ and let $\chi \in \Gamma_{\text {top }}$, i.e.

$$
\chi \circ \varphi(x)=h(T x) / h(x)
$$

for some continuous function $h: X \rightarrow \mathbb{S}^{1}$. We define $w: X \times G \rightarrow \mathbb{S}^{1}$ :

$$
w(x, g)=h(x)^{-1} \chi(g) .
$$

This function is continuous and $T_{\varphi}$-invariant, whence it is constant on each minimal component. It follows that $h(x)^{-1} \chi(g) \chi\left(g_{0}\right)=w\left(x, g g_{0}\right)=w(x, g)=$ $h(x)^{-1} \chi(g)$, which implies $\chi\left(g_{0}\right)=1$. Therefore $g_{0} \in F_{\text {top }}$.

Suppose now that there exists $g_{0} \in F_{\text {top }} \backslash H_{\text {top }}$. Then there exists $\chi \in \widehat{G}$ such that $\chi\left(g_{0}\right) \neq 1$ and $\chi\left(H_{\text {top }}\right)=\{1\}$. Let $\tau: X \rightarrow G / H_{\text {top }}$ satisfy (61). It follows that $\chi \circ \tau$ is well-defined and we obtain

$$
\chi \circ \tau(T x)=\chi \circ \varphi(x) \cdot \chi \circ \tau(x)
$$

which implies

$$
\chi \circ \varphi(x)=\frac{\chi \circ \tau(T x)}{\chi \circ \tau(x)},
$$

i.e. $\chi \in \Gamma_{\text {top }}$ and consequently $\chi\left(g_{0}\right)=1$ which is a contradiction.

Relation between the ergodic and the minimal components Let $T$ be a minimal homeomorphism of a compact metric space $X$ and let $\mu$ be a $T$-invariant probability Borel measure, ergodic with respect to $T$. Let $\varphi: X \rightarrow G$ be continuous. Then $T_{\varphi}$ is a homeomorphism of $X \times G$ and $T_{\varphi} \in \operatorname{Aut}\left(X \times G, \mathcal{B} \otimes \mathcal{B}(G), \mu \otimes \lambda_{G}\right)$. Let $\lambda \in \mathcal{P}\left(T_{\varphi}, \mathcal{B}, \mu\right)$. There are two natural partitions associated to $T_{\varphi}$ : 
- $\mathcal{P}_{\text {erg }}$ is the partition into the ergodic components of $T_{\varphi}$,

- $\mathcal{P}_{\min }$ is the partition into the minimal components of $T_{\varphi}$.

Partition $\mathcal{P}_{\text {erg }}$ is clearly measurable.

Proposition 2.5.13 Partition $\mathcal{P}_{\text {min }}$ is measurable.

Proof Let $M \subset X \times G$ be as in Lemma 2.5.11, in particular, $M$ is a minimal component of $T_{\varphi}$. Let $s: G / H_{t o p} \rightarrow G$ be a Borel selector of the canonical projection $\pi: G \rightarrow$ $G / H_{\text {top }}$. Consider $\eta: X \rightarrow G$ given by $\eta=s \circ \tau$. We obtain

$$
\varphi^{\prime}(x):=\varphi(x) \eta(x)(\eta(T x))^{-1} \in H_{t o p}
$$

and

$$
M=\bigcup_{x \in X}\{x\} \times\left(\eta(x) H_{t o p}\right)
$$

so the map

$$
(x, h) \mapsto(x, \eta(x) h)
$$

settles an equivariant Borel isomorphism of $X \times H$ (considered with $T_{\varphi^{\prime}}$ ) and $M$ (considered with $T_{\varphi}$ ). Moreover, (62) can be naturally extended to a Borel isomorphism of $X \times H_{\text {top }} \times G / H_{\text {top }}$ and $X \times G$. Clearly the partition of $X \times H_{\text {top }} \times G / H_{\text {top }}$ given by relevant translations of $X \times H_{t o p} \times\{1\}$ (indexed by $G / H_{t o p}$ ) is measurable for the product measure $\mu \otimes \lambda_{H_{t o p}} \otimes \lambda_{G / H_{t o p}}$. Hence its image by the Borel extensions of (62) is also a measurable partition for the image of the measure $\mu \otimes \lambda_{H_{t o p}} \otimes \lambda_{G / H_{\text {top }}}$. This image is equal to $\mu \otimes \lambda_{G}$, so the partition into minimal components is indeed measurable.

Remark 2.5.14 Since the partition into the ergodic components can be defined as the finest measurable partition whose atoms are invariant under the action of the homeomorphism in question, $\mathcal{P}_{\text {erg }}$ is finer than $\mathcal{P}_{\text {min }}$.

As a direct consequence of the above remark we obtain the following:

Remark 2.5.15 We have $H \supset H_{t o p}$. The condition $H=H_{t o p}$ is necessary and sufficient for the ergodic components of $T_{\varphi}$ to be the same as its minimal components. Moreover, $H=H_{t o p}$ if and only if $\Gamma=\Gamma_{\text {top }}$.

\section{Unique ergodicity}

Proposition 2.5.16 [Furstenberg, see the proof of Theorem I.4 in [12] and Proposition 3.10 in [13]] Let $T: X \rightarrow X$ be uniquely ergodic and let $\varphi: X \rightarrow G$ be a continuous cocycle with values in a compact Abelian group. If $T_{\varphi}$ is ergodic with respect to $\mu \otimes \lambda_{G}$ then it is uniquely ergodic. 


\section{A remark on the KBSZ criterion for $T x=x+\alpha$}

Let $T x=x+\alpha$ be an irrational rotation on $\mathbb{T}$.

Proposition 2.5.17 Let $A \subset \mathbb{Z}$.

1. If $A$ is such that

$$
r A \cap s A=\emptyset \text { for sufficiently large prime numbers } r \neq s,
$$

then (7) holds true for every $f \in C(\mathbb{T})$ such that supp $\widehat{f}:=\{n \in \mathbb{Z}: \widehat{f}(n) \neq$ $0\}=A$.

2. Suppose that A contains infinitely many primes. Let $f \in C(\mathbb{T})$ be such that supp $\widehat{f}=A$ and all nonzero Fourier coefficients are positive. Then (7) fails for $f$.

Remark 2.5.18 Note that every finite set satisfies (63), so all trigonometric polynomials satisfy (7); the set $\left\{2^{n}: n \geq 1\right\}$ is an example of an infinite set satisfying (63).

Proof of Proposition 2.5.17 We will consider the behavior of the sums in $(7)$ at $(0,0)$. Given two different prime numbers $r, s$, we set $I_{r, s}:=\{(x, y): s x=r y\}$, which is a closed subgroup of $\mathbb{T}^{2}$ (of course $(0,0) \in I_{r, s}$ ), invariant under $T^{r} \times T^{s}$. It is not hard to see that

$$
\frac{1}{N} \sum_{n \leq N} \delta_{r n \alpha, s n \alpha} \rightarrow \lambda_{I_{r, s}}
$$

and that

$$
\begin{aligned}
& W: I_{r, s} \rightarrow \mathbb{T}, W(x, y)=a x+b y(a r+b s=1) \\
& \text { is a continuous group isomorphism. }
\end{aligned}
$$

In particular, $W$ sends $\lambda_{I_{r, s}}$ to $\lambda_{\mathbb{T}}$. In view of (64),

$$
\frac{1}{N} \sum_{n \leq N} f\left(T^{r n} 0\right) \overline{f\left(T^{s n} 0\right)} \rightarrow \int_{I_{r, s}} f \otimes \bar{f} d \lambda_{I_{r, s}}
$$

Since $W^{-1}(t)=(r t, s t)$, it follows that

$$
\int_{I_{r, s}} f \otimes \bar{f} d \lambda_{I_{r, s}}=\int_{\mathbb{T}}(f \otimes \bar{f}) \circ W^{-1}(t) d t=\int_{\mathbb{T}} f(r t) \overline{f(s t)} d t .
$$

Now, if $f(t)=\sum_{n \in \mathbb{Z}} c_{n} e^{2 \pi i n t}$ then

$$
f(r t)=\sum_{n \in \mathbb{Z}} c_{n} n e^{2 \pi i r n t}, \quad f(s t)=\sum_{n \in \mathbb{Z}} c_{n} e^{2 \pi i s n t}
$$

and we can compute $\int_{I_{r, s}} f \otimes \bar{f} d \lambda_{I_{r, s}}$ using (66), (67) and Parseval's formula. 
1. Fix infinitely many pairs $\left(r_{j}, s_{j}\right) \in A \times A$ of distinct prime numbers, $r_{j}, s_{j} \rightarrow \infty$. Take $j \geq 1$. It is enough to show that $\int_{I_{r_{j}, s_{j}}} f \otimes \bar{f} d \lambda_{I_{r_{j}, s_{j}}} \neq 0$. By Parseval's formula, and the fact that all Fourier coefficients of $f$ are positive, we have $\int_{I_{r_{j}, s_{j}}} f \otimes \bar{f} d \lambda_{I_{r_{j}, s_{j}}} \geq 0$. Since $r_{j} s_{j}=s_{j} r_{j}$, the $s_{j} r_{j}$ th Fourier coefficient of $f\left(r_{j} \cdot\right)$ is $c_{s_{j}}>0$, while the $r_{j} s_{j}$ th Fourier coefficient of $f\left(s_{j} \cdot\right)$ is $c_{r_{j}}>0$. Hence, by Parseval's formula, $\int_{I_{r_{j}, s_{j}}} f \otimes \bar{f} d \lambda_{I_{r_{j}, s_{j}}}>0$.

2. Each $(x, y) \in \mathbb{T}^{2}$ belongs to a coset of $I_{r, s}$. The proof for an arbitrary coset of $I_{r, s}$ goes along the same lines as for $I_{r, s}$ itself (on the cosets of $I_{r, s}$, we consider translations of Haar measure $\lambda_{I_{r, s}}$ and $W$ is practically the same). Since $r A \cap s A=$ $\emptyset$, the supports of the Fourier transforms of $f(r \cdot)$ and $f(s \cdot)$ are disjoint, whence $\int_{\mathbb{T}} f(r t) \overline{f(s t)} d t=0$.

\section{References}

1. El Abdalaoui, E.H., Kasjan, S., Lemańczyk, M.: 0-1 Sequences of the Thue-Morse type and Sarnak's conjecture. arXiv:1304.3587 (To appear in Proc. Amer. Math. Soc)

2. El Abdalaoui, E.H., Lemańczyk, M., de la Rue, T.: On spectral disjointness of powers for rank-one transformations and Möbius orthogonality. J. Funct. Anal. 266, 284-317 (2014)

3. Anzai, H.: Ergodic skew product transformations on the torus. Osaka Math. J. 3, 83-99 (1951)

4. Auslander, J.: On the proximal relation in topological dynamics. Proc. Amer. Math. Soc. 11, 890-895 (1960)

5. Bourgain, J.: Möbius-Walsh correlation bounds and an estimate of Mauduit and Rivat. J. Anal. Math. 119, 147-163 (2013)

6. Bourgain, J.: On the correlation of the Moebius function with rank-one systems. J. Anal. Math. 120, 105-130 (2013)

7. Bourgain, J., Sarnak, P., Ziegler, T.: Disjointness of Möbius from horocycle flows. In: From Fourier analysis and number theory to radon transforms and geometry, Dev. Math., vol. 28, pp. 67-83. Springer, New York (2013)

8. Dartyge, C., Tenenbaum, G.: Sommes des chiffres de multiples d'entiers. Ann. Inst. Fourier (Grenoble) 55, 2423-2474 (2005)

9. Davenport, H.: On some infinite series involving arithmetical functions. II. Quart. J. Math. Oxford, 8, 313-320 (1937)

10. Ellis, R.: A semigroup associated with a transformation group. Trans. Amer. Math. Soc. 94, 272-281 (1960)

11. Furstenberg, H.: Strict ergodicity and transformation of the torus. Am. J. Math. 83, 573-601 (1961)

12. Furstenberg, H.: Disjointness in ergodic theory, minimal sets, and a problem in Diophantine approximation. Math. Syst. Theory 1, 1-49 (1967)

13. Furstenberg, H.: Recurrence in ergodic theory and combinatorial number theory. Princeton University Press, Princeton (1981) (M. B. Porter Lectures)

14. Green, B.: On (not) computing the Möbius function using bounded depth circuits. Combin. Probab. Comput. 21, 942-951 (2012)

15. Green, B., Tao, T.: The Möbius function is strongly orthogonal to nilsequences. Ann. of Math. 175(2), 541-566 (2012)

16. Indlekofer, K.-H., Kátai, I.: Investigations in the theory of $q$-additive and $q$-multiplicative functions. I. Acta Math. Hungar. 91, 53-78 (2001)

17. Kátai, I.: A remark on a theorem of H. Daboussi. Acta Math. Hungar. 47, 223-225 (1986)

18. Katok, A.: Combinatorial constructions in ergodic theory and dynamics. University Lecture Series, vol. 30. American Mathematical Society, Providence, RI (2003)

19. Keane, M.: Generalized Morse sequences. Z. Wahrscheinlichkeitstheorie und Verw. Gebiete 10, 335353 (1968)

20. Khinchin, A.Y.: Continued fractions. Dover Publications Inc., Mineola (1997) (Reprint of the 1964 translation) 
21. Kwiatkowski, J., Lemańczyk, M., Rudolph, D.: A class of real cocycles having an analytic coboundary modification. Israel J. Math. 87, 337-360 (1994)

22. Lemańczyk, M., Mentzen, M.K.: Compact subgroups in the centralizer of natural factors of an ergodic group extension of a rotation determine all factors. Ergodic Theory Dynam. Syst. 10, 763-776 (1990)

23. Liu, J., Sarnak, P.: The Möbius function and distal flows. arXiv:1303.4957 (2013)

24. Mauduit, C., Rivat, J.: Sur un problème de Gelfond: la somme des chiffres des nombres premiers. Ann. Math. 171(2), 1591-1646 (2010)

25. Mauduit, C., Rivat, J.: Prime numbers along Rudin-Shapiro sequences. J. Eur. Math. Soc. (to appear)

26. Sarnak, P.: Mobius randomness and dynamics. Not. S. Afr. Math. Soc. 43, 89-97 (2012)

27. Siemaszko, A.: Ergodic and topological properties of distal systems. Ph.D. thesis (1996) 